\title{
Minimal Siegel modular threefolds
}

\author{
BY VALERI GRITSENKO $\dagger$ \\ St. Petersburg Department Steklov Mathematical Institute, \\ Fontanka 27, 191011 St Petersburg, Russia \\ e-mail: gritsenk@cfgauss.uni-math.gwdg.de; gritsenk@pdmi.ras.ru \\ AND KLAUS HULEK \\ Institut für Mathematik, Universität Hannover, Postfach 6009, \\ D-30060 Hannover, Germany \\ e-mail: hulek@math.uni-hannover.de \\ (Received 7 May 1996; revised 9 September 1996)
}

\begin{abstract}
The starting point of this paper is the maximal extension $\Gamma_{t}^{*}$ of $\Gamma_{t}$, the subgroup of $\mathrm{Sp}_{4}(\mathbb{Q})$ which is conjugate to the paramodular group. Correspondingly we call the quotient $\mathscr{A}_{t}^{*}=\Gamma_{t}^{*} \backslash \mathbb{H}_{2}$ the minimal Siegel modular threefold. The space $\mathscr{A}_{t}^{*}$ and the intermediate spaces between $\mathscr{A}_{t}=\Gamma_{t} \backslash \mathbb{H}_{2}$ which is the space of $(1, t)$-polarized abelian surfaces and $\mathscr{A}_{t}^{*}$ have not yet been studied in any detail. Using the Torelli theorem we first prove that $\mathscr{A}_{t}^{*}$ can be interpreted as the space of Kummer surfaces of $(1, t)$ polarized abelian surfaces and that a certain degree 2 quotient of $\mathscr{A}_{t}$ which lies over $\mathscr{A}_{t}^{*}$ is a moduli space of lattice polarized $K 3$ surfaces. Using the action of $\Gamma_{t}^{*}$ on the space of Jacobi forms we show that many spaces between $\mathscr{A}_{t}$ and $\mathscr{A}_{t}^{*}$ possess a nontrivial 3-form, i.e. the Kodaira dimension of these spaces is non-negative. It seems a difficult problem to compute the Kodaira dimension of the spaces $\mathscr{A}_{t}^{*}$ themselves. As a first necessary step in this direction we determine the divisorial part of the ramification locus of the finite map $\mathscr{A}_{t} \rightarrow \mathscr{A}_{t}^{*}$. This is a union of Humbert surfaces which can be interpreted as Hilbert modular surfaces.
\end{abstract}

\section{Introduction}

The moduli space $\mathscr{A}_{t}$ of abelian surfaces with a $(1, t)$-polarization is the quotient of the Siegel upper half plane $\mathbb{H}_{2}$ by a subgroup $\Gamma_{t}$ of $\operatorname{Sp}_{4}(\mathbb{Q})$ which is conjugate to the paramodular group $\widetilde{\Gamma}_{t}$. In Section 1 we define an isomorphism between the symplectic group and the special orthogonal group $\mathrm{SO}(3,2)$ over the integers.

This exhibits $\Gamma_{t} /\left\{ \pm E_{4}\right\}$ as a subgroup of the orthogonal group $\mathrm{SO}\left(L_{t}\right)$ where $L_{t}$ is the lattice of rank 5 equipped with the form $<2 t>\oplus 2 U$ (here $U$ denotes the hyperbolic plane). Let $\widehat{L}_{t}$ be the dual lattice of $L_{t}$. The image of $\Gamma_{t}$ in $\mathrm{O}\left(L_{t}\right)$ acts trivially on $\widehat{L}_{t} / L_{t}$. The orthogonal group $\mathrm{O}\left(\widehat{L}_{t} / L_{t}\right)$ is isomorphic to $(\mathbb{Z} / 2 \mathbb{Z})^{\nu(t)}$ where

$\dagger$ Supported by Institute Fourier, Grenoble, the Schwerpunktprogramm 'Komplexe Mannigfaltigkeiten' grant Hu 337/2-4 and DFG grant 436 Rus 17/108/95. 
$\nu(t)$ is the number of prime divisors of $t$. For every $d|| t$ (i.e. $d \mid t$ and $(d, t / d)=1$ ) we construct an element $V_{d}$ in $\mathrm{Sp}_{4}(\mathbb{R})$. These elements $V_{d}$ define a normal extension $\Gamma_{t}^{*}$ of $\Gamma_{t}$ of index $2^{\nu(t)}$ such that $\Gamma_{t}^{*} / \Gamma_{t} \cong \mathrm{O}\left(\widehat{L}_{t} / L_{t}\right)$. It turns out that $\Gamma_{t}^{*}$ is the maximal normal extension of $\Gamma_{t}$ as a discrete subgroup of $\mathrm{Sp}_{4}(\mathbb{R})$. Hence we can consider the moduli spaces $\mathscr{A}_{t}^{*}=\Gamma_{t}^{*} \backslash \mathbb{H}_{2}$ as 'minimal' Siegel modular threefolds. In Section 1 we also give a geometric interpretation of the action of $V_{d}$ on the moduli space $\mathscr{A}_{t}$. In particular $V_{t}$ identifies a polarized abelian surface with its dual. It turns out that the space $\left(\Gamma_{t} \cup \Gamma_{t} V_{t}\right) \backslash \mathbb{H}_{2}$ is isomorphic to the moduli space of lattice polarized $K 3$ surfaces with a polarization of type $<2 t>\oplus 2 E_{8}(-1)$. Lattice polarized $K 3$ surfaces have been studied by Nikulin [N2]. They play a role in mirror symmetry for $K 3$ surfaces (see Dolgachev $[\mathbf{D}]$ ). We also prove that the variety $\mathscr{A}_{t}^{*}$ itself is the space of Kummer surfaces associated to $(1, t)$-polarized abelian surfaces. (For a precise statement see Theorem 1.5.) In particular, this implies that a $(1, t)$-polarized abelian surface and its dual have isomorphic Kummer surfaces. Surprisingly, this result seems not to have been noticed before. At this point one can ask the question which of the Siegel modular varieties lying between $\mathscr{A}_{t}$ and $\mathscr{A}_{t}^{*}$ are moduli spaces of $K 3$ surfaces.

In Section 2 we study the action of the elements $V_{d}$ on the space of Jacobi forms. It turns out that this gives rise to a decomposition of the space of Jacobi forms which was originally found by Eichler and Zagier [EZ]. Using lifting results due to the first author this enables us to prove that many moduli spaces lying between $\mathscr{A}_{t}$ and the minimal Siegel modular threefold $\mathscr{A}_{t}^{*}$ are not unirational, resp. have nonnegative Kodaira dimension. This method, however, unfortunately does not give us information about the geometric genus or the Kodaira dimension of $\mathscr{A}_{t}^{*}$ itself. To determine these seems a fairly hard problem. We hope to come back to it. The general philosophy behind these considerations is the question where these moduli spaces fit into the classification theory of algebraic threefolds. Very little is known about this. For example one can ask, whether the fact that $\mathscr{A}_{t}^{*}$ is minimal (in the sense described above) for square free $t$ is in any way reflected in terms of the classification theory of threefolds. To prove results about the Kodaira dimension of the spaces $\mathscr{A}_{t}^{*}$ requires a good knowledge of the singularities of these spaces which is a hard problem in itself. Since the singularities of $\mathscr{A}_{t}$ are known (cf. [B]) the problem is equivalent to determining the ramification locus of the finite map $\mathscr{A}_{t} \rightarrow \mathscr{A}_{t}^{*}$. As a first step we compute the divisorial part of this ramification locus for square free $t$ (the general case can be treated by the same method). This can be shown to be a finite union of Humbert surfaces. To determine these we reexamine the theory of Humbert surfaces from the point of view of the orthogonal group, which turns out to be a very useful way of studying Humbert surfaces. An example, originally due to Brasch, shows that the ramification locus can also contain curve components. We finally interpret the Humbert surfaces in the ramification locus as Hilbert modular surfaces.

\section{The symplectic and orthogonal groups}

The local isomorphism between the symplectic group $\mathrm{Sp}_{4}(\mathbb{R})$ and the special orthogonal group $\mathrm{SO}(3,2)_{\mathbb{R}}$ of signature $(3,2)$ is well known. In this section we define this isomorphism over $\mathbb{Z}$.

Let us fix a lattice

$$
L=e_{1} \mathbb{Z} \oplus e_{2} \mathbb{Z} \oplus e_{3} \mathbb{Z} \oplus e_{4} \mathbb{Z}
$$


We identify $l \in L$ with a column-vector in the basis $\left\{e_{i}\right\} . L^{2}=L \wedge L$ is the lattice of integral bivectors, which is isomorphic to the lattice of integral skew-symmetric matrices. The bivector $e_{i} \wedge e_{j}$ corresponds to the elementary skew-symmetric matrix $E_{i j}$, which has only two non-zero elements $e_{i j}=1$ and $e_{j i}=-1$. Any linear transformation $g: L \rightarrow L$ induces a linear map $\wedge^{2} g: L \wedge L \rightarrow L \wedge L$ on the $\mathbb{Z}$-lattice of bivectors. If $g$ is represented with respect to the basis $\left\{e_{i}\right\}$ by the matrix $G$, then

$$
\left(\wedge^{2} g\right)(X)=G X^{t} G \quad \text { for any } \quad X=\sum_{i<j} x_{i j} e_{i} \wedge e_{j} \in L \wedge L .
$$

One can define a symmetric bilinear form $(X, Y)$ on $L \wedge L$

$$
X \wedge Y=(X, Y) e_{1} \wedge e_{2} \wedge e_{3} \wedge e_{4} \in \wedge^{4} L .
$$

It is known, that $(X, X)=2 \operatorname{Pf}(X)$, where $\operatorname{Pf}(X)$ is the Pfaffian of the matrix $X$, and $\operatorname{Pf}\left(M X^{t} M\right)=\operatorname{Pf}(X) \operatorname{det} M$.

Definition. The group

$$
\widetilde{\Gamma}_{t}=\left\{g: L \rightarrow L \mid \wedge^{2} g\left(W_{t}\right)=W_{t}, \quad \text { where } \quad W_{t}=e_{1} \wedge e_{3}+t e_{2} \wedge e_{4}\right\}
$$

is called the integral paramodular group of level $t$.

The lattice $L_{t}=W_{t}^{\perp}$ consisting of all elements of $L \wedge L$ orthogonal to $W_{t}$ has the following basis

$$
L_{t}=\left(e_{1} \wedge e_{2}, e_{2} \wedge e_{3}, e_{1} \wedge e_{3}-t e_{2} \wedge e_{4}, e_{4} \wedge e_{1}, e_{4} \wedge e_{3}\right) \mathbb{Z}^{5} .
$$

We fix this basis for the rest of the paper. The symmetric bilinear form $(\cdot, \cdot)$ defines a quadratic form $S$ of signature $(3,2)$ on the lattice $L_{t}$, which has the following form in the given basis

$$
S_{t}=\left(\begin{array}{ccccc}
0 & 0 & 0 & 0 & -1 \\
0 & 0 & 0 & -1 & 0 \\
0 & 0 & 2 t & 0 & 0 \\
0 & -1 & 0 & 0 & 0 \\
-1 & 0 & 0 & 0 & 0
\end{array}\right) .
$$

The group of the real points of the paramodular group is conjugate to $\mathrm{Sp}_{4}(\mathbb{R})$. Thus the determinant of any element of the paramodular group equals one and $\wedge^{2} g$ keeps the bilinear form on $L \wedge L$.

This gives us a homomorphism from the symplectic group in the orthogonal group of the isometries of the lattice $L_{t}$

$$
\wedge^{2}: \widetilde{\Gamma}_{t} \rightarrow \mathrm{O}\left(L_{t}\right)
$$

The paramodular group $\widetilde{\Gamma}_{t}$ is conjugate to a subgroup of the usual rational symplectic group:

$$
\Gamma_{t}:=I_{t}^{-1} \widetilde{\Gamma}_{t} I_{t}=\left\{\left(\begin{array}{cccc}
* & * & * & t * \\
t * & * & t * & t * \\
* & * & * & t * \\
* & t^{-1} * & * & *
\end{array}\right) \in \operatorname{Sp}_{4}(\mathbb{Q})\right\},
$$

where all entries $*$ denote integers and $I_{t}=\operatorname{diag}(1,1,1, t)$. 
The quotient space

$$
\mathscr{A}_{t}=\Gamma_{t} \backslash \mathbb{H}_{2}
$$

is the coarse moduli space of abelian surfaces with a polarization of type $(1, t)$.

The composition of the conjugation with the homomorphism $\wedge^{2}$ defines a homomorphism

$$
\Psi: \Gamma_{t} \rightarrow \mathrm{O}\left(L_{t}\right) \quad \text { where } \quad \Psi(g)=\wedge^{2}\left(I_{t} g I_{t}^{-1}\right) .
$$

One can extend $\Psi$ to the real symplectic group $\Gamma_{t}(\mathbb{R}) \cong \operatorname{Sp}_{4}(\mathbb{R})$.

Let $\widehat{L}_{t}=\left\{u \in L_{t} \otimes \mathbb{Q} \mid \forall l \in L_{t}(l, u) \in \mathbb{Z}\right\}$ be the dual lattice of $L_{t}$. The discriminant group

$$
A_{t}:=\widehat{L}_{t} / L_{t}=(2 t)^{-1} \mathbb{Z} / \mathbb{Z} \cong \mathbb{Z} / 2 t \mathbb{Z}
$$

is a finite abelian group equipped with a quadratic form

$$
q_{t}: A_{t} \times A_{t} \rightarrow(2 t)^{-1} \mathbb{Z} / 2 \mathbb{Z} \quad q_{t}(l, l) \equiv(l, l)_{\widehat{L}_{t}} \bmod 2 \mathbb{Z}
$$

(see [N1] for a general definition). Any $g \in \mathrm{O}\left(L_{t}\right)$ acts on the finite group $A_{t}$. By

$$
\widehat{\mathrm{O}}\left(L_{t}\right)=\left\{g \in \mathrm{O}\left(L_{t}\right) \mid \forall \ell \in \widehat{L}_{t} \quad g \ell-\ell \in L_{t}\right\}
$$

we denote the subgroup of the orthogonal group consisting of elements which act identically on the discriminant group.

One can easily prove the next lemma (see $[$ G1]).

Lemma $1 \cdot 1$. The following relations are valid

1. $\Psi\left(\Gamma_{t}\right) \subset \widehat{\mathrm{SO}}\left(L_{t}\right)=\widehat{\mathrm{O}}\left(L_{t}\right) \cap \mathrm{SO}\left(L_{t}\right)$;

2. $\operatorname{Ker} \Psi=\left\{ \pm E_{4}\right\}$.

The finite orthogonal group $\mathrm{O}\left(A_{t}\right)$ can be described as follows. For every $d \| t$ (i.e. $d \mid t$ and $(d, t / d)=1)$ there exists a unique $(\bmod 2 t)$ integer $\xi_{d}$ satisfying

$$
\xi_{d}=-1 \bmod 2 d, \quad \xi_{d}=1 \bmod 2 t / d .
$$

All such $\xi_{d}$ form the group

$$
\Xi(t)=\left\{\xi \bmod 2 t \mid \xi^{2}=1 \bmod 4 t\right\} \cong(\mathbb{Z} / 2 \mathbb{Z})^{\nu(t)},
$$

where $\nu(t)$ is the number of prime divisors of $t$. It is evident that $\mathrm{O}\left(A_{t}\right) \cong \Xi(t)$.

One can take an element in $\mathrm{SO}\left(L_{t}\right)$ realising the multiplication by $\xi_{d}$ on $A_{t}$. It gives us an element in $\mathrm{Sp}_{4}(\mathbb{R})$ with integral $\Psi$-image. For example, for every $d \| t$ we can define $x, y \in \mathbb{Z}$ (which are not uniquely determined) such that

$$
x d-y t_{d}=1 \quad \text { where } t_{d}=\frac{t}{d} .
$$

The matrix

$$
\widetilde{V}_{d}=\left(\begin{array}{cccc}
d x & -1 & 0 & 0 \\
-y t & d & 0 & 0 \\
0 & 0 & d & y t \\
0 & 0 & 1 & d x
\end{array}\right)
$$


is an integral symplectic similitude of degree $d$. We put

$$
V_{d}=\frac{1}{\sqrt{ } d} \widetilde{V}_{d} \in \mathrm{Sp}_{4}(\mathbb{R})
$$

$V_{d}$ has the following $\Psi$-image

$$
\Psi\left(V_{d}\right)=\left(\begin{array}{ccccc}
1 & 0 & 0 & 0 & 0 \\
0 & d & -2 y t & y^{2} t_{d} & 0 \\
0 & -1 & d x+t_{d} y & -x y & 0 \\
0 & t_{d} & -2 t x & x^{2} d & 0 \\
0 & 0 & 0 & 0 & 1
\end{array}\right)
$$

We note here that

$$
x d+t_{d} y=-1 \bmod 2 d \text { and } x d+t_{d} y=1 \bmod 2 t_{d},
$$

thus $V_{d}$ induces the multiplication by $\xi_{d}$ on $A_{t}$.

It is easy to see, that for all $V_{d}(d|| t)$

$$
V_{d}^{2} \in \Gamma_{t}, \quad V_{d} \Gamma_{t} V_{d}=\Gamma_{t},
$$

i.e. the $V_{d}$ are involutions modulo $\Gamma_{t}$. Therefore one can define the following normal extension of the paramodular group $\Gamma_{t}$.

Definition. $\Gamma_{t}^{*}$ is the group generated by the elements of $\Gamma_{t}$ and $V_{d}$ for all $d \| t$.

In accordance with Lemma 1.1 any element in $\Psi\left(V_{d} \Gamma_{t}\right)$ defines the same automorphism of $A_{t}$, thus

$$
\Gamma_{t}^{*} / \Gamma_{t} \cong \mathrm{O}\left(\widehat{L}_{t} / L_{t}\right) \cong \Xi(t) \cong(\mathbb{Z} / 2 \mathbb{Z})^{\nu(t)} .
$$

The real orthogonal group $\mathrm{O}_{\mathbb{R}}\left(L_{t}\right)=\mathrm{O}\left(L_{t} \otimes \mathbb{R}\right)$ acts on a domain lying on a projective quadric, more exactly on

$$
\mathbb{P H}_{t}^{3}=\mathbb{P H}_{L_{t}}^{3}=\left\{Z \in \mathbb{P}\left(L_{t} \otimes \mathbb{C}\right) \mid(Z, Z)=0,(Z, \bar{Z})<0\right\}=\mathbb{P H}_{t}^{+} \cup \overline{\mathbb{P H}}_{t}^{+},
$$

where

$$
\mathbb{P H}_{t}^{+}=\left\{Z={ }^{t}\left(\left(t z_{2}^{2}-z_{1} z_{3}\right), z_{3}, z_{2}, z_{1}, 1\right) \cdot z_{0} \in \mathbb{P H}_{t}^{3} \mid \operatorname{Im}\left(z_{1}\right)>0\right\} .
$$

This is a classical homogeneous domain of type IV. The condition $(Z, \bar{Z})<0$ is equivalent to

$$
y_{1} y_{3}-t y_{2}^{2}>0 \quad \text { where } y_{i}=\operatorname{Im}\left(z_{i}\right) .
$$

Taking $z_{0}=1$ one gets the corresponding cylindric domain in the affine coordinates $\left(z_{i}\right)_{1 \leqslant i \leqslant 3}$

$$
\mathbb{H}_{t}^{+}=\left\{Z={ }^{t}\left(z_{3}, z_{2}, z_{1}\right) \in \mathbb{C}^{3} \mid y_{1} y_{3}-t y_{2}^{2}>0, \quad y_{1}=\operatorname{Im}\left(z_{1}\right)>0\right\} .
$$

The domain $\mathbb{H}_{t}^{+}$for $t=1$ coincides with the Siegel upper half-plane $\mathbb{H}_{2}$. For a general $t$ one can define the following isomorphism of the complex domains

$$
\psi_{t}: \mathbb{H}_{2} \rightarrow \mathbb{H}_{t}^{+} \quad \psi_{t}\left(\begin{array}{cc}
\tau_{1} & \tau_{2} \\
\tau_{2} & \tau_{3}
\end{array}\right)={ }^{t}\left(\frac{\tau_{3}}{t}, \frac{\tau_{2}}{t}, \tau_{1}\right) .
$$

The linear action of the real orthogonal group $\mathrm{O}_{\mathbb{R}}\left(L_{t}\right)$ on $\mathbb{P H}_{t}^{+}$defines 'fractionallinear' transformations on $\mathbb{H}_{t}^{+}$. $\mathrm{By} \mathrm{O}_{\mathbb{R}}^{+}\left(L_{t}\right)$ we denote the subgroup of index 2 of 
the orthogonal group consisting of elements which leave $\mathbb{P H}_{t}^{+}$invariant. (This is the subgroup of the elements with real spin norm equal one.)

Proposition 1.2. Let $t$ be square free. Then $\Psi$ defines the following isomorphisms

$$
\Psi: \Gamma_{t}^{*} /\left\{ \pm E_{4}\right\} \rightarrow \mathrm{SO}^{+}\left(L_{t}\right),
$$

where $\mathrm{SO}^{+}\left(L_{t}\right)=\operatorname{SO}\left(L_{t}\right) \cap \mathrm{O}_{\mathbb{R}}^{+}\left(L_{t}\right)$, and

$$
\Psi: \Gamma_{t} /\left\{ \pm E_{4}\right\} \rightarrow \widehat{\mathrm{SO}}^{+}\left(L_{t}\right),
$$

where $\widehat{\mathrm{SO}}^{+}\left(L_{t}\right)=\mathrm{SO}^{+}\left(L_{t}\right) \cap \widehat{\mathrm{SO}}\left(L_{t}\right)$. Moreover the following diagram is commutative

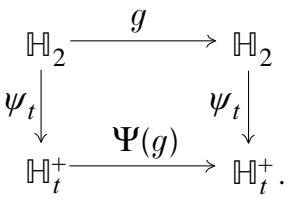

Proof. The diagram is commutative for any $g \in \mathrm{Sp}_{4}(\mathbb{R})$. To prove this one has to calculate the images of standard generators of $\mathrm{Sp}_{4}(\mathbb{R})$ under $\Psi$.

It is known that for square free $t$, the group $\mathrm{P} \Gamma_{t}^{*} \cong \Gamma_{t}^{*} /\left\{ \pm E_{4}\right\}$ is a maximal discrete subgroup of the group of analytic automorphisms of $\mathbb{H}_{2}$ and $\left[\Gamma_{t}^{*}: \Gamma_{t}\right]=2^{\nu(t)}$ (see for example $[\mathbf{A l}],[\mathbf{G u}]$ ). From the description of the finite orthogonal group $\mathrm{O}\left(A_{t}\right)$ given in (1.4) we obtain that $\left[\mathrm{SO}^{+}\left(L_{t}\right): \widehat{\mathrm{SO}}^{+}\left(L_{t}\right)\right]=2^{\nu(t)}$. The statement of the proposition about the isomorphism of the groups follows from the maximality of $\Gamma_{t}^{*}$ and Lemma 1.1.

The coset $V_{t} \Gamma_{t}$ (in the case $d=t$ we may take $x=0, y=-1$ ) can also be written in the form

$$
V_{t} \Gamma_{t}=\left(\begin{array}{cccc}
0 & \sqrt{ } t^{-1} & 0 & 0 \\
\sqrt{ } t & 0 & 0 & 0 \\
0 & 0 & 0 & \sqrt{ } t \\
0 & 0 & \sqrt{ } t^{-1} & 0
\end{array}\right) \Gamma_{t} .
$$

According to $(1 \cdot 3)$ and $(1 \cdot 5) \Psi\left(V_{t}\right)$ defines the multiplication by -1 on $\widehat{L}_{t} / L_{t}$, i.e. $V_{t}$ corresponds to the element $\xi_{t}=-1$ of $\Xi(t)$ (see 1.4). Therefore

$$
-\Psi\left(V_{t}\right) \in \widehat{\mathrm{O}}\left(L_{t}\right) \text {. }
$$

Elements $M$ and $-M \in \mathrm{O}\left(L_{t}\right)$ define the same transformation of the domain $\mathbb{H}_{t}^{+}$. Thus we have

Corollary 1·3. Let t be square free. The groups

$$
\Psi\left(\Gamma_{t} \cup \Gamma_{t} V_{t}\right) \quad \text { and } \quad \mathrm{O}^{*}\left(L_{t}\right)=\widehat{\mathrm{O}}\left(L_{t}\right) \cap \mathrm{O}_{\mathbb{R}}^{+}\left(L_{t}\right)
$$

coincide, if we consider them as groups of analytic transformations of $\mathbb{P H}_{t}^{+}$.

Proposition 1.4. The quotient

$$
\left(\Gamma_{t} \cup \Gamma_{t} V_{t}\right) \backslash \mathbb{H}_{2}
$$

is isomorphic to the moduli space of polarized K3 surfaces with a polarization of type $<2 t>\oplus 2 E_{8}(-1)$. 
Proof. It is known that a moduli space of polarized $K 3$ surfaces is a quotient of a 19-dimensional homogeneous domain of type IV by an arithmetic group. In the proposition we consider polarized $K 3$ surfaces with a condition on the Picard group or equivalently on the lattice of its transcendental cycles. To formulate these conditions we need some definitions (see [N2], [D]).

Let $X$ be a $K 3$ surface. Let us take a sublattice $D_{t}=<2 t>\oplus E_{8}(-1) \oplus E_{8}(-1)$ of the lattice

$$
L_{K 3}=U \oplus U \oplus U \oplus E_{8}(-1) \oplus E_{8}(-1) \cong H^{2}(X, \mathbb{Z}),
$$

where $\langle 2 t\rangle(t \in \mathbb{Z})$ denotes the one-dimensional lattice generated by a vector $l$ such that $l^{2}=2 t, U$ is the hyperbolic plane with quadratic form $\left(\begin{array}{ll}0 & 1 \\ 1 & 0\end{array}\right)$ and $E_{8}(-1)$ is the even unimodular lattice of dimension 8 with the negative definite quadratic form. We note that $\operatorname{sign}\left(D_{t}\right)=(1,16)$ and $D_{t}^{\perp} \cong U \oplus U \oplus<-2 t>=L_{t}^{\prime}=L_{t}(-1)$. (Notation $L_{t}(-1)$ means that we multiply the quadratic form $S_{t}$ on the lattice $L_{t}$ by -1.) We recall (see $[\mathbf{N 1}]$ ) that the orthogonal group

$$
\mathrm{O}\left(D_{t}, L_{K 3}\right)=\left\{g: L_{K 3} \rightarrow L_{K 3}|g|_{D_{t}} \equiv \mathrm{id}\right\}
$$

is isomorphic to the group

$$
\widehat{\mathrm{O}}\left(L_{t}^{\prime}\right)=\left\{g: L_{t}^{\prime} \rightarrow L_{t}^{\prime} \mid \forall l \in \widehat{L}_{t}^{\prime} g l-l \in L_{t}^{\prime}\right\} .
$$

A marked $D_{t}$-polarized $K 3$-surface is defined by the following datum (see [N2] and [D] for more details): a surface $X$, a fundamental domain $C(M)^{+}$of a group generated by some 2-reflections of the lattice $D_{t}$ acting on a connected component $V\left(D_{t}\right)^{+}$of the cone $V\left(D_{t}\right)=\left\{v \in D_{t} \otimes \mathbb{R} \mid(v, v)>0\right\}$ and an isomorphism of the lattices $\phi: H^{2}(X, \mathbb{Z}) \rightarrow L_{K 3}$, such that $\phi^{-1}\left(D_{t}\right) \subset \operatorname{Pic}(X), \phi^{-1}\left(V\left(D_{t}\right)^{+}\right) \subset V(X)^{+}$and $\phi^{-1}\left(C(M)^{+}\right)$contains at least one numerically effective divisor class. By $V(X)^{+}$one denotes the connected component of the cone

$$
V(X)=\left\{v \in H_{\mathbb{R}}^{1,1}(X) \mid(v, v)>0\right\}
$$

containing the cohomology class of a Kähler form on $X$.

Let us denote by $\omega_{X}$ a holomorphic 2 -form which generates $H^{2,0}(X)$. Its image under the isometry $\phi$ belongs to the following domain in the projective space $\mathbb{P}^{4}$

$$
\phi\left(\omega_{X}\right) \in \mathscr{D}_{m}=\left\{v \in \mathbb{P}\left(L_{t}^{\prime} \otimes \mathbb{C}\right):(v, v)=0, \quad(v, \bar{v})>0\right\} .
$$

This domain is an example of the domains of type IV. Its connected components are isomorphic to the domain $\mathbb{H}_{t}^{+}$introduced after $(1 \cdot 7)$.

The quotient

$$
\mathscr{M}\left(<2 t>\oplus 2 E_{8}(-1)\right)=\mathrm{O}^{*}\left(L_{t}^{\prime}\right) \backslash \mathbb{H}_{t}^{+}
$$

is the moduli space of isomorphism classes of $<2 t>\oplus 2 E_{8}(-1)$-polarized $K 3$ surfaces. In accordance with Corollary 1.3

$$
\mathscr{M}\left(<2 t>\oplus 2 E_{8}(-1)\right) \cong\left(\Gamma_{t} \cup \Gamma_{t} V_{t}\right) \backslash \mathbb{H}_{2} .
$$

We now want to explain the relationship between the variety $\mathscr{A}_{t}^{*}=\Gamma_{t}^{*} \backslash \mathbb{H}_{2}$ and the space of Kummer surfaces associated to $(1, t)$-polarized abelian surfaces. For an abelian surface $A$ we denote by $X=\operatorname{Km}(A)$ its associated Kummer surface.

Theorem 1.5. (i) Let $A, A^{\prime}$ be two $(1, t)$-polarized abelian surfaces which define the same point in $\mathscr{A}_{t}^{*}$. Then their Kummer surfaces $X, X^{\prime}$ are isomorphic. 
(ii) Assume that the Neron-Severi group of $A$, resp. $A^{\prime}$ is generated by the polarization. Then the converse is true: If $A$ and $A^{\prime}$ have isomorphic Kummer surfaces, then $A$ and $A^{\prime}$ define the same point in $\mathscr{A}_{t}^{*}$.

Proof. We consider the commutative diagram

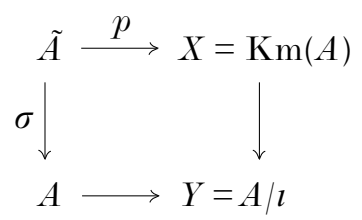

where $\tilde{A}$ is the blow-up of $A$ in the 16 points of order 2 . By [BPV, p. 246] the map

$$
\alpha=p_{!} \circ \sigma^{*}: H^{2}(A, \mathbb{Z}) \rightarrow H^{2}(X, \mathbb{Z})
$$

multiplies the intersection form by 2 and hence is in particular a monomorphism. Let $T \subset H^{2}(X, \mathbb{Z})$ be the orthogonal complement of the sublattice of $H^{2}(X, \mathbb{Z})$ generated by the polarization and the nodal classes. Using $[\mathbf{B P V}$, corollary VIII $\cdot 5 \cdot 6]$ it follows that $T \cong 2 U(2) \oplus<-4 t>$. If $A$ is generic, i.e. the Neron-Severi group is generated by the polarization, then $T$ is the transcendental lattice of $X$.

(i) Assume that $A$ and $A^{\prime}$ define the same point in $\mathscr{A}_{t}^{*}$. Then there exists an element $V$ in $\Gamma_{t}^{*}$ which induces an isomorphism of the lattices $T$ and $T^{\prime}$ of $X$ and $X^{\prime}$ respectively. By the Torelli theorem for Kummer surfaces it now suffices to prove the

Claim. The isomorphism $V: T \rightarrow T^{\prime}$ can be extended to a Hodge isometry $\widetilde{V}: H^{2}(X, \mathbb{Z}) \rightarrow H^{2}\left(X^{\prime}, \mathbb{Z}\right)$.

Proof of claim. By definition $T$ is a primitive non-degenerate sublattice of the unimodular lattice $H^{2}(X, \mathbb{Z})$. Its orthogonal complement $T^{\perp}$ in $H^{2}(X, \mathbb{Z})$ is an even indefinite lattice of signature $(1,16)$. The discriminant groups $A_{T}$ and $A_{T^{\perp}}$ are isomorphic and have five generators. By [N1, theorem 1.14.2] the homomorphism $\mathrm{O}\left(T^{\perp}\right) \rightarrow \mathrm{O}\left(A_{T^{\perp}}, q_{T^{\perp}}\right)$ is surjective. Using [N1, corollary 1.5.2] one can, therefore, construct an extension of the isomorphism $V$.

(ii) An isomorphism $f: X \rightarrow X^{\prime}$ induces an isomorphism of transcendental lattices. Under the assumption stated this defines an isometry $V: T \rightarrow T^{\prime}$ where $T \cong T^{\prime} \cong$ $2 U(2) \oplus<-4 t>=L_{t}(-2)$, which is an element of the group $\mathrm{O}^{+}\left(L_{t}(-2)\right)$. Since $\mathrm{O}^{+}\left(L_{t}(-2)\right)=\mathrm{O}^{+}\left(L_{t}\right)$ it follows from Proposition 1.2 that there is an element $\bar{V} \in \Gamma_{t}^{*}$ which defines one of the elements $\pm V$. The element $\bar{V}$ identifies the points defined by $A$ and $A^{\prime}$ resp.

Remark. The above theorem justifies it to consider $\mathscr{A}_{t}^{*}$ as the moduli space of Kummer surfaces associated to abelian surfaces with a $(1, t)$-polarization.

Remark. In [D, example 6.5] Dolgachev considered the space of $M_{t}$-polarized abelian surfaces where $M_{t}$ is the orthogonal complement of $2 U(2) \oplus<-4 t>$ in $L_{K 3}$. This leads to a subgroup of finite index of $\mathrm{O}^{+}\left(L_{t}\right)$ and hence a covering space of $\mathscr{A}_{t}^{*}$.

Our next aim is to interpret the involutions $V_{d}$ geometrically. Because of Proposition $1 \cdot 2$ the element $V_{d}$ induces a map from $\mathscr{A}_{t}^{*}$ to itself. 
Let $(A, H)$ be a $(1, t)$-polarized abelian surface. The polarization $H$ defines an isogeny

$$
\begin{aligned}
\lambda_{H}: & A \rightarrow \widehat{A}=\mathrm{Pic}^{0} A \\
& x \mapsto T_{x}^{*} \mathscr{L} \otimes \mathscr{L}^{-1}
\end{aligned}
$$

where $\mathscr{L}$ is a line bundle representing $H$ and $T_{x}$ denotes translation by $x$. The map $\lambda_{H}$ only depends on $H$, not on the line bundle $\mathscr{L}$. There is a (non-canonical) isomorphism ker $\lambda_{H} \cong \mathbb{Z}_{t} \times \mathbb{Z}_{t}$. For every divisor $d$ of $t$ there is a unique subgroup $G(d) \subset \operatorname{ker} \lambda_{H}$ which is isomorphic to $\mathbb{Z}_{d} \times \mathbb{Z}_{d}$. This subgroup defines a quotient

$$
\lambda_{d}: \quad A \rightarrow A / G(d)=A^{\prime} .
$$

If $A$ is given by the period matrix

$$
\Omega=\left(\begin{array}{cccc}
1 & 0 & \tau_{1} & \tau_{2} \\
0 & t & \tau_{2} & \tau_{3}
\end{array}\right), \quad \tau=\left(\begin{array}{cc}
\tau_{1} & \tau_{2} \\
\tau_{2} & \tau_{3}
\end{array}\right) \in \mathbb{H}_{2}
$$

then $A^{\prime}$ is given by

$$
\Omega^{\prime}=\left(\begin{array}{cccc}
d & 0 & d \tau_{1} & \tau_{2} \\
0 & t_{d} & \tau_{2} & \tau_{3} / d
\end{array}\right)
$$

The abelian surface $A^{\prime}$ carries a uniquely determined polarization $H^{\prime}$ with

$$
d H=\lambda_{d}^{*}\left(H^{\prime}\right) .
$$

The polarization $H^{\prime}$ is of type $e \cdot\left(1, t / e^{2}\right)$ where $e=\left(d, t_{d}\right)$. Altogether this shows that we have a morphism of moduli spaces

$$
\begin{aligned}
\Phi=\Phi(d): \mathscr{A}_{t} & \rightarrow \mathscr{A}_{t / e^{2}} \\
(A, H) & \mapsto\left(A^{\prime}, H^{\prime}\right) .
\end{aligned}
$$

If $d=t$ we obtain as a special case the map

$$
\begin{aligned}
\Phi(t): \mathscr{A}_{t} & \rightarrow \mathscr{A}_{t} \\
(A, H) & \mapsto(\widehat{A}, \widehat{H})
\end{aligned}
$$

which maps an abelian surface to its dual polarized abelian surface.

Proposition 1·6. Let $d$ be a divisor of $t$ with $\left(d, t_{d}\right)=1$. Then the map

$$
\Phi(d): \mathscr{A}_{t} \rightarrow \mathscr{A}_{t}
$$

is the map induced by $V_{d}$.

Proof. For $\tau=\left(\begin{array}{cc}\tau_{1} & \tau_{2} \\ \tau_{2} & \tau_{3}\end{array}\right) \in \mathbb{H}_{2}$ we have the following formula for the action

$$
V_{d}<\tau>=\left(\begin{array}{cc}
x & -1 \\
-y t_{d} & d
\end{array}\right)\left(\begin{array}{cc}
d \tau_{1} & \tau_{2} \\
\tau_{2} & \tau_{3} / d
\end{array}\right)\left(\begin{array}{cc}
x & -y t_{d} \\
-1 & d
\end{array}\right) .
$$

Now consider the matrix

$$
\left(\begin{array}{cccc}
1 & t_{d} & 0 & 0 \\
y & x d & 0 & 0 \\
0 & 0 & x & -y t_{d} \\
0 & 0 & -1 & d
\end{array}\right) \in \mathrm{SL}_{4}(\mathbb{Z})
$$


This matrix transforms the symplectic form $d e_{1} \wedge e_{3}+t_{d} e_{2} \wedge e_{4}$ into $W_{t}$ (see (1.1)). The claim now follows from the equality

$$
\left(\begin{array}{cc}
x & -1 \\
-y t_{d} & d
\end{array}\right)\left(\begin{array}{cccc}
d & 0 & d \tau_{1} & \tau_{2} \\
0 & t_{d} & \tau_{2} & \tau_{3} / d
\end{array}\right)\left(\begin{array}{cccc}
1 & t_{d} & 0 & 0 \\
y & x d & 0 & 0 \\
0 & 0 & x & -y t_{d} \\
0 & 0 & -1 & d
\end{array}\right)=\left(\begin{array}{ccc}
1 & 0 & \\
0 & t & V_{d}<\tau>
\end{array}\right)
$$

Remark. In view of Theorem 1.5 this shows in particular that a $(1, t)$-polarized abelian surface and its dual polarized abelian surface have isomorphic Kummer surfaces. Surprisingly this seems not to have been noticed before.

\section{Nonunirationality of some quotient spaces}

We have already shown that the Siegel modular threefolds $\mathscr{A}_{t} /\left\langle V_{t}\right\rangle$ and $\mathscr{A}_{t}^{*}$ are moduli spaces of $K 3$ surfaces. There are $2^{\nu(t)}-2$ other threefolds between $\mathscr{A}_{t}$ and $\mathscr{A}_{t}^{*}$. Let us take for example two primes $p \neq q$ and let $t=p q$. The involutions $V_{p}$ and $V_{q}$ give rise to the following moduli spaces

$$
\mathscr{A}_{p q}^{(p)}=\mathscr{A}_{p q} /<V_{p}>, \mathscr{A}_{p q}^{(q)}=\mathscr{A}_{p q} /<V_{q}>, \mathscr{A}_{p q}^{*}=\mathscr{A}_{p q} /<V_{p}, V_{q}>=\Gamma_{p q}^{*} \backslash \mathbb{H}_{2}
$$

resp. a commutative diagram

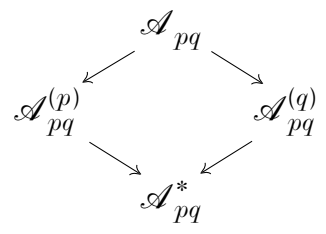

where all maps are 2:1. Using the modular forms constructed in $[\mathbf{G 1}]$ we can obtain information about the geometric genus of some of these moduli spaces.

By $J_{k, t}^{\text {cusp }}$ we denote the space of Jacobi cusp forms of weight $k$ and index $t$. In [G1] a lifting was constructed which associates to a Jacobi form $\Phi \in J_{k, t}^{\text {cusp }}$ a cusp form $F_{\Phi} \in \mathscr{M}_{k}\left(\widehat{\Gamma}_{t}\right)$ of weight $k$ with respect to the group

$$
\widehat{\Gamma}_{t}=\left\{\left(\begin{array}{cccc}
* & t * & * & * \\
* & * & * & * t^{-1} \\
* & t * & * & * \\
t * & t * & t * & *
\end{array}\right) \in \operatorname{Sp}_{4}(\mathbb{Q})\right\},
$$

where all entries $*$ denote integers.

The groups $\Gamma_{t}$ and $\widehat{\Gamma}_{t}$ are conjugate. Indeed if $C_{t}=\operatorname{diag}\left(1, t^{-1}, 1, t\right)$ then $\widehat{\Gamma}_{t}=$ $C_{t} \Gamma_{t} C_{t}^{-1}$. For

$$
\widehat{V}_{t}={ }^{t} V_{t}=\left(\begin{array}{cccc}
0 & \sqrt{ } t & 0 & 0 \\
\sqrt{ } t^{-1} & 0 & 0 & 0 \\
0 & 0 & 0 & \sqrt{ } t^{-1} \\
0 & 0 & \sqrt{ } t & 0
\end{array}\right)
$$

it was proved in $[\mathbf{G} \mathbf{1}$, formula $(2 \cdot 8)]$ that

$$
F_{\Phi}(Z)=\left.(-1)^{k} F_{\Phi}\right|_{k} \widehat{V}_{t}(Z) \text {. }
$$


or equivalently

$$
F_{\Phi}\left(\begin{array}{cc}
\tau_{1} & \tau_{2} \\
\tau_{2} & \tau_{3}
\end{array}\right)=F_{\Phi}\left(\begin{array}{cc}
t \tau_{3} & \tau_{2} \\
\tau_{2} & t^{-1} \tau_{1}
\end{array}\right)
$$

In this section we describe the behaviour of $F_{\Phi}$ with respect to the group

$$
\widehat{\Gamma}_{t}^{*}=<\widehat{\Gamma}_{t}, \widehat{V}_{d} \mid d \| t>
$$

where $\widehat{V}_{d}=C_{t} V_{d} C_{t}^{-1}$.

Eichler and Zagier [EZ, section 5], have constructed a decomposition of the space of Jacobi forms

$$
J_{k, t}^{\text {cusp }}=\bigoplus_{\epsilon} J_{k, t}^{\epsilon},
$$

where $\epsilon$ runs over all characters of the group

$$
\Xi(t)=\left\{\xi \bmod 2 t \mid \xi^{2} \equiv 1 \bmod 4 t\right\} \cong(\mathbb{Z} / 2 \mathbb{Z})^{\nu(t)}
$$

satisfying $\epsilon(-1)=(-1)^{k}$. For any $d$ with $d \| t$ one can define an operator $W_{d}$ acting on $J_{k, t}$ in the following way [EZ, section 5]. For

$$
\Phi\left(\tau_{1}, \tau_{2}\right)=\sum_{\substack{n, l \in \mathbb{Z} \\ 4 n t>l^{2}}} f(n, l) \exp \left(2 \pi i\left(n \tau_{1}+l \tau_{2}\right)\right) \in J_{k, t}^{\text {cusp }},
$$

we put

$$
\left(\Phi \mid W_{d}\right)\left(\tau_{1}, \tau_{2}\right)=\sum_{\substack{n, l \in \mathbb{Z} \\ 4 n t>l^{2}}} f\left(n^{\prime}, l^{\prime}\right) \exp \left(2 \pi i\left(n \tau_{1}+l \tau_{2}\right)\right) \in J_{k, t}^{\text {cusp }},
$$

where $l^{\prime}, n^{\prime}$ are determined by

$$
l^{\prime} \equiv-l \bmod 2 d, \quad l^{\prime} \equiv l \bmod 2 t / d, \quad 4 n^{\prime} t-l^{2}=4 n t-l^{2} .
$$

All $W_{d}$ are involutions. They form a group isomorphic to $\Xi(t)$. The subspaces $J_{k, t}^{\epsilon}$ are eigenspaces of the operation $W_{d}$, namely

$$
J_{k, t}^{\epsilon}=\left\{\Phi \in J_{k, t}^{\text {cusp }}|\Phi| W_{d}=\epsilon\left(W_{d}\right) \Phi\right\} .
$$

Note that if

$$
\Phi\left(\tau_{1}, \tau_{2}\right)=\sum_{\mu \bmod 2 t} \varphi_{\mu}\left(\tau_{1}\right) \theta_{t, \mu}\left(\tau_{1}, \tau_{2}\right) \in J_{k, t}^{\epsilon}
$$

is the standard decomposition of the Jacobi form $\Phi$ with respect to the thetafunctions $\theta_{t, \mu}\left(\tau_{1}, \tau_{2}\right)$ then for $\xi_{d} \in \boldsymbol{\Xi}(t)$

$$
\varphi_{\xi_{d} \mu}\left(\tau_{1}\right)=\epsilon\left(\xi_{d}\right) \varphi_{\mu}\left(\tau_{1}\right) .
$$

Theorem 2-1. Let $\Phi \in J_{k, t}^{\epsilon}$ be a Jacobi form and $F_{\Phi} \in \mathscr{M}_{k}\left(\widehat{\Gamma}_{t}\right)$ be its lifting. For any divisor $d$ of $t$ with $\left(d, t_{d}\right)=1$ the following equality holds

$$
\left.F_{\Phi}\right|_{k} \widehat{V}_{d}=\epsilon\left(\xi_{d}\right) F_{\Phi} .
$$

Proof. Let us recall the definition of the lifting $F_{\Phi}$ in terms of the Fourier expansion [G2]. If

$$
\Phi\left(\tau_{1}, \tau_{2}\right)=\sum_{\substack{n, l \in \mathbb{Z} \\ 4 n t>l^{2}}} f(n, l) \exp \left(2 \pi i\left(n \tau_{1}+l \tau_{2}\right)\right) \in J_{k, t}^{\text {cusp }}
$$


then

$$
F_{\Phi}(Z)=\sum_{N \in \mathfrak{I}_{t}} b(N) \exp (2 \pi i \operatorname{tr}(N Z)),
$$

where summation is taken over all positive definite symmetric matrices of the following form

$$
N \in \mathfrak{A}_{t}=\left\{\left(\begin{array}{cc}
n & l / 2 \\
l / 2 & m t
\end{array}\right)>0 \mid n, l, m \in \mathbb{Z}\right\}
$$

and

$$
b\left(\begin{array}{cc}
n & l / 2 \\
l / 2 & m t
\end{array}\right)=\sum_{a \mid(n, l, m)} a^{k-1} f\left(\frac{n m}{a^{2}}, \frac{l}{a}\right) .
$$

The action of $\widehat{V}_{d}$ on $F_{\Phi}$ is given by

$$
\left(\left.F_{\Phi}\right|_{k} \widehat{V}_{d}\right)(Z)=F_{\Phi}\left(d^{-1} A_{d} Z^{t} A_{d}\right) \quad \text { where } A_{d}=\left(\begin{array}{cc}
d x & -t \\
-y & d
\end{array}\right)
$$

or

$$
\begin{aligned}
\left(\left.F_{\Phi}\right|_{k} \widehat{V}_{d}\right)(Z) & =\sum_{N \in \mathfrak{Q}_{t}} b(N) \exp \left(2 \pi i \operatorname{tr}\left(d^{-1}{ }^{t} A_{d} N A_{d} Z\right)\right) \\
& =\sum_{N \in \mathfrak{H}_{t}} b\left(d^{-1}{ }^{t} \tilde{A}_{d} N \tilde{A}_{d}\right) \exp (2 \pi i \operatorname{tr}(N Z))
\end{aligned}
$$

where $\tilde{A}_{d}=d A_{d}^{-1}=\left(\begin{array}{cc}d & t \\ y & d x\end{array}\right)$. Let $\widetilde{N}=d^{-1}{ }^{t} \tilde{A}_{d} N \tilde{A}_{d}=\left(\begin{array}{cc}\tilde{n} & \tilde{l} / 2 \\ \tilde{l} / 2 & \tilde{m} t\end{array}\right)$. Clearly $\operatorname{det} N=$ $\operatorname{det} \widetilde{N}$. It is easy to see that the elements $n, l, m$ and $\tilde{n}, \tilde{l}, \widetilde{m}$ have the same set of common divisors. Moreover

$$
\tilde{l}=l\left(y t_{d}+d x\right)+2(n t+x y m t) \quad \text { and } \quad \tilde{l} \equiv\left\{\begin{array}{rr}
-l & \bmod 2 d \\
l & \bmod 2 t_{d} .
\end{array}\right.
$$

Hence, by the definition of $J_{k, t}^{\epsilon}$ we have

$$
b\left(d^{-1 t} \tilde{A}_{d} N \tilde{A}_{d}\right)=\sum_{a \mid(n, l, m)} a^{k-1} f\left(\frac{\tilde{n} \tilde{m}}{a^{2}}, \frac{\tilde{l}}{a}\right)=\epsilon\left(\xi_{d}\right) b(N)
$$

which proves the theorem.

This result has some implications on the Kodaira dimension of Siegel modular varieties which are quotients of $\mathscr{A}_{t}$. This question has not yet been studied. We consider the following results as a first step towards a more systematic study of the Kodaira dimension of these varieties. Whenever we speak of the Kodaira dimension of some moduli space $\mathscr{A}$ we mean the Kodaira dimension of a desingularization of a projective compactification of $\mathscr{A}$.

Corollary 2.2. Let $p \neq q$ be primes $\geqslant 5$. Then the Kodaira dimension of at least one of the spaces $\mathscr{A}_{p q}^{(p)}$ or $\mathscr{A}_{p q}^{(q)}$ is $\geqslant 0$.

Proof. The Eichler-Zagier decomposition gives a decomposition

$$
J_{3, p q}=J_{3, p q}^{(+,-)} \oplus J_{3, p q}^{(-,+)} .
$$

If $p, q \geqslant 5$ then there exists a cusp form in $J_{3, p q}$ and hence in $J_{3, p q}^{(+,-)}$or $J_{3, p q}^{(-,+)}$. By 
[G1], [G2] this can be lifted to a weight 3 cusp form with respect to $\left\langle\Gamma_{t}, V_{p}\right\rangle$, resp. $<\Gamma_{t}, V_{q}>$. By Freitag's extension theorem this defines a differential form on any desingularization of a projective compactification of $\mathscr{A}_{p q}^{(p)}$, resp. $\mathscr{A}_{p q}^{(q)}$. This gives the result.

For any integer $t$ let us take a character of the group $\Xi(t)$ isomorphic to the orthogonal group of the discriminant group of $L_{t}$ (see (1.4))

$$
\epsilon: \Xi(t) \rightarrow\{ \pm 1\}
$$

We define a set $U(\epsilon)=\left\{V_{d} \in \Gamma_{t}^{*} \mid \epsilon\left(\xi_{d}\right)=1\right\}$ and a subgroup

$$
\Gamma_{t}^{\epsilon}=<\Gamma_{t}, \xi_{d} \mid \xi_{d} \in U(\epsilon)>\subset \Gamma_{t}^{*}
$$

of $\Gamma_{t}^{*}$. Theorem $2 \cdot 1$ and the method of the proof of Corollary $2 \cdot 2$ gives us the next result.

Corollary 2.3. If $\operatorname{dim}\left(J_{3, t}^{\epsilon}\right)>0$, then the Kodaira dimension of the quotient space

$$
\mathscr{A}_{t}^{\epsilon}=\Gamma_{t}^{\epsilon} \backslash \mathbb{H}_{2}
$$

is nonnegative.

Remark. Corollary $2 \cdot 3$ gives information about $\mathscr{A}_{t}^{\epsilon}$ only if $V_{t} \notin U(\epsilon)$. If $\epsilon\left(V_{t}\right)=$ $\epsilon(-1)=1$, then $\operatorname{dim}\left(J_{3, t}^{\epsilon}\right)=0$.

Corollary 2.4. Let $t \geqslant 21(t \neq 24,30,36)$ and let its number of prime divisors $\nu(t) \geqslant 2$. Then there exists a finite quotient of $\mathscr{A}_{t}$ of degree $2^{\nu(t)-1}$ which is not unirational.

Proof. For any integer $t$ from the corollary the dimension of $J_{3, t}^{\text {cusp }}$ is positive. Thus there is a character $\epsilon$ of $\Xi(t)$ such that $\epsilon\left(\xi_{t}\right)=-1$ and $\operatorname{dim} J_{3, t}^{\epsilon}>0 . \Xi(t) \cong(\mathbb{Z} / 2 \mathbb{Z})^{\nu(t)}$ therefore $\left[\Gamma_{t}^{\epsilon}: \Gamma_{t}\right]=2^{\nu(t)-1}$.

Using dimension formulae for the spaces $J_{k, t}^{\epsilon}$ one can obtain more precise results. It is easy to get an exact dimension formula using the trace formula of the operator $W_{d}$ on the space $J_{k, t}^{\text {cusp }}$ given in $[\mathbf{S Z}]$. By definition of $W_{d}$ we have

$$
\operatorname{tr}\left(W_{d}, J_{3, t}^{\text {cusp }}\right)=\sum_{\epsilon} \epsilon\left(\xi_{d}\right) \operatorname{dim}\left(J_{3, t}^{\epsilon}\right),
$$

where the sum is taken over all characters of $\Xi(t)$. Therefore

$$
\operatorname{dim}\left(J_{3, t}^{\epsilon}\right)=2^{-\nu(t)} \sum_{d \| t} \epsilon\left(\xi_{d}\right) \operatorname{tr}\left(W_{d}, J_{3, t}^{\text {cusp }}\right) .
$$

For weight 3 the trace formula of $W_{d}$ on $J_{3, t}^{\text {cusp }}$ (we recall that $d \mid t$ and $\left(d, t_{d}\right)=1$, where $\left.t_{d}=t / d\right)$ proved in $[\mathbf{S Z}$, theorem 1] can be reduced to the following expression

$$
\begin{aligned}
\operatorname{tr}\left(W_{d}, J_{3, t}^{\text {cusp }}\right)= & \frac{1}{4} \sum_{e \mid d} H_{t_{d}}(-4 e)-\frac{1}{4} \sum_{e^{\prime} \mid t_{d}} H_{d}\left(-4 e^{\prime}\right)+\frac{3}{2}\left(H_{d}(0)-H_{t_{d}}(0)\right) \\
& +\frac{1}{2}\left(\delta_{2}\left(t_{d}\right) H_{d}(-4)-\delta_{2}(d) H_{t_{d}}(-4)\right)+\left(\delta_{3}\left(t_{d}\right) H_{d}(-3)-\delta_{3}(d) H_{t_{d}}(-3)\right) \\
& +\frac{1}{4}\left(\left(Q\left(t_{d}\right), 2\right) Q(d)-(Q(d), 2) Q\left(t_{d}\right)\right) .
\end{aligned}
$$

We denote by $Q(n)$ the greatest integer whose square divides $n ; \delta_{a}(b)=1$ or 0 if $a \mid b$ 
or $a \nmid b$ and $H_{n}(\Delta)$ is a generalization of the Hurwitz-Kronecker class number, i.e $H_{1}(0)=-\frac{1}{12}$ and $H_{1}(\Delta)$ for $\Delta<0$ is the number of equivalence classes with respect to $\mathrm{SL}_{2}(\mathbb{Z})$ of integral, positive definite, binary quadratic forms of discriminant $\Delta$, counting forms equivalent to a multiple of $x^{2}+y^{2}$ (resp. $x^{2}+x y+y^{2}$ ) with multiplicity $\frac{1}{2}\left(\operatorname{resp} . \frac{1}{3}\right)$. For $n \geqslant 2$ with $(n, \Delta)=a^{2} b$ and square free $b$

$$
H_{n}(\Delta)= \begin{cases}a^{2} b\left(\frac{\Delta / a^{2} b^{2}}{n / a^{2} b}\right) H_{1}\left(\Delta / a^{2} b^{2}\right) & \text { if } a^{2} b^{2} \mid \Delta \\ 0 & \text { otherwise }\end{cases}
$$

where $(:)$ is the generalized Kronecker symbol.

We note that the trace formula has the simplest form for a square free $t$ coprime to 6 :

$$
\operatorname{tr}\left(W_{d}, J_{3, t}^{\text {cusp }}\right)=\frac{1}{4}\left(\sum_{e \mid d}\left(\frac{-4 e}{t_{d}}\right) H_{1}(-4 e)-\sum_{e^{\prime} \mid t_{d}}\left(\frac{-4 e^{\prime}}{d}\right) H_{1}\left(-4 e^{\prime}\right)\right)+\frac{t_{d}-d}{8} .
$$

Example $\mathbf{2 \cdot 5}$. The calculation gives us only thirteen different threefolds of type $\mathscr{A}_{t}^{\epsilon}$ with $t$ having only two prime divisors $\left(t=p^{a} q^{b}\right)$, whose geometric genus could be equal to zero. They correspond to the following trivial subspaces of $J_{3, t}^{\text {cusp }}$ of type $J_{3, p^{a} q^{b}}^{-+}$(this notation means that $J_{3, p^{a} q^{b}}^{-+}=J_{3, p^{a} q^{b}}^{\epsilon}$ with $\epsilon\left(\xi_{p^{a}}\right)=-1$ and $\epsilon\left(\xi_{q^{b}}\right)=1$ ):

$$
\begin{array}{ccccccc}
J_{3,2.11}^{-+}, & J_{3,2.13}^{-+}, & J_{3,2.17}^{-+}, & J_{3,2.19}^{-+}, & J_{3,2.25}^{-+}, & J_{3,2.27}^{-+}, \\
J_{3,3.7}^{-+}, & J_{3,3.13}^{-+}, & J_{3,3.16}^{-+}, & J_{3,5.7}^{-+}, & J_{3,5.8}^{-+}, & J_{3,7.4}^{-+}, & J_{3,7.8}^{-+}
\end{array}
$$

We may add to this list twenty threefolds $\mathscr{A}_{t}$ with $t=1, \ldots, 12,14,15,16,18,20,24$, 30, 36 (see [G2]) whose geometric genus could be zero. According to classical results and new results of M. Gross and S. Popescu (see $[\mathbf{G P}]$ ) it really is for $t=1, \ldots, 12$, 14, 16, 18, 20.

Example $2 \cdot 6$. We have the following quotients of order 4 and 8 which are not unirational. One has

$$
\operatorname{dim} J_{3,42}^{\text {cusp }}=\operatorname{dim} J_{3,2.3 .7}^{++-}=1 .
$$

Thus for $\mathscr{A}_{42}^{(2,3)}=<V_{2}, V_{3}>\backslash \mathscr{A}_{42}$ we obtain $h^{3,0}\left(\mathscr{A}_{42}^{(2,3)}\right) \geqslant 1$.

The geometric genus of all four threefolds of type $<V_{a}, V_{b}, V_{c}>\backslash \mathscr{A}_{210}$, where $a, b, c \in\{2,3,5,7\}$ is positive.

\section{The Humbert surfaces and the ramification locus}

If one wants to compute the Kodaira dimension of the variety $\mathscr{A}_{t}^{*}=\Gamma_{t}^{*} \backslash \mathbb{H}_{2}$ it is essential to know its singularities. Already the singularities of $\mathscr{A}_{t}$ are very complicated to determine (cf. [B]). In view of this result the corresponding result for $\mathscr{A}_{t}^{*}$ is essentially equivalent to a description of the ramification locus of the finite map $\mathscr{A}_{t} \rightarrow \mathscr{A}_{t}^{*}$, i.e. the locus where the stabilizer of the finite group $\Gamma_{t}^{*} / \Gamma_{t}$ is not trivial. This, too, turns out to be a difficult question. As a first step we shall determine the divisorial part of the ramification locus which is a union of a finite number of Humbert surfaces. We shall restrict ourselves to $t$ square free, but our method works in general. Here we study Humbert surfaces from the point of view of the orthogonal group, rather than the symplectic group, which turns out to be a very appropriate approach. 
First we collect some known facts about divisors on the homogeneous domain $\mathbb{P H}_{t}^{+}$. For any $v \in L_{t} \otimes \mathbb{R}$ we set

$$
\mathscr{H}_{v}=\left\{Z \in \mathbb{P H}_{t}^{+} \mid v \cdot Z=0\right\},
$$

where $v \cdot u=(v, u)_{t}$ is the bilinear product corresponding to the quadratic form $S_{t}$ $($ see $(1 \cdot 2))$.

\section{LEMMa $3 \cdot 1$.}

1. $\mathscr{H}_{g v}=g^{-1} \mathscr{H}_{v}$ for any $g \in \mathrm{O}_{\mathbb{R}}^{+}\left(L_{t}\right)$.

2. Let $v \neq 0$, then $\mathscr{H}_{v} \neq \varnothing$ if and only if $v^{2}>0$.

3. $\mathscr{H}_{v} \cap \mathscr{H}_{u} \neq \varnothing$ if and only if the matrix $\left(\begin{array}{cc}v^{2} & v . u \\ v . u & u^{2}\end{array}\right)$ is positive definite.

Proof. 1. The first property is trivial.

2. The orthogonal group $\mathrm{O}_{\mathbb{R}}^{+}\left(L_{t}\right)$ acts transitively on $\mathbb{P H}_{t}^{+}$. Thus any $Z \in \mathbb{P H}_{t}^{+}$can be reduced to $Z_{\mathbf{i}}={ }^{t}(1, i, 0, i, 1)$ in the coordinates $\left(z_{i}\right)$ from $1 \cdot 7$. If $v=(a, b, c, d, e)$ and $v \cdot Z_{\mathbf{i}}=0$, then $a=-e$ and $b=-d$. Thus $v^{2}=2 a^{2}+2 b^{2}+2 t c^{2}>0$.

Let $L_{t} \otimes \mathbb{R}=\mathbb{R} v \oplus V$ with $v^{2}>0$. One has $\operatorname{sign}(V)=(2,2)$. The group $\mathrm{SO}^{+}(V) \cong$ $\mathrm{SO}_{\mathbb{R}}^{+}(2,2)$ is locally isomorphic to $\mathrm{SL}_{2}(\mathbb{R}) \times \mathrm{SL}_{2}(\mathbb{R})$. Thus

$$
\mathscr{H}_{v} \cong \mathbb{P H}_{V}^{+} \cong \mathbb{H}_{1} \oplus \mathbb{H}_{1},
$$

where $\mathbb{H}_{1}$ is the usual upper half-plane. This proves the second statement.

3. Let us suppose that $\mathscr{H}_{v} \cap \mathscr{H}_{u} \neq \varnothing$. It follows from $(x u-v)^{2}>0$ that the matrix in 3 is positive definite.

If the symmetric bilinear form on the plane $P=\mathbb{R} v \oplus \mathbb{R} u$ is positive definite, then $\operatorname{sign}\left(P^{\perp}\right)=(1,2)$. The group $\mathrm{SO}^{+}\left(P^{\perp}\right) \cong \mathrm{SO}^{+}(1,2)_{\mathbb{R}}$ is locally isomorphic to $\mathrm{SL}_{2}(\mathbb{R})$ and

$$
\mathscr{H}_{v} \cap \mathscr{H}_{u} \cong \mathbb{P H}_{P^{\perp}}^{+} \cong \mathbb{H}_{1} .
$$

Remark. For $l \in L_{t}\left(l^{2}>0\right)$ the group $\widehat{\mathrm{SO}}^{+}\left(l^{\perp}\right)$ is isomorphic to a subgroup of $\mathrm{SL}_{2}(\mathbb{Z}) \times \mathrm{SL}_{2}(\mathbb{Z})$ or to a subgroup of a Hilbert modular group.

Definition. Let $\ell \in \widehat{L}_{t}$ be a vector in the dual lattice. The Humbert surface $H_{\ell}$ is defined by

$$
H_{\ell}=\pi\left(\bigcup_{g \in \widehat{S O}^{+}\left(L_{t}\right)} \mathscr{H}_{g \ell}\right),
$$

where $\pi: \mathbb{P H}_{t}^{+} \rightarrow \widehat{\mathrm{SO}}^{+}\left(L_{t}\right) \backslash \mathbb{P H}_{t}^{+}$is the natural projection.

$\mathscr{H}_{\ell}$ depends only on the one dimensional lattice $\mathbb{Z} \ell$, thus we can restrict ourselves to primitive vectors $\ell \in \widehat{L}_{t}$. The primitivity means that $\ell / d \notin \widehat{L}_{t}$ for any integer $d>1$. The first statement of Lemma $3 \cdot 1$ says that there is a one to one correspondence between the $\widehat{\mathrm{SO}}^{+}\left(L_{t}\right)$-orbits of primitive vectors $\ell \in \widehat{L}_{t}$ with positive norm and the Humbert surfaces.

It is well known that for any even integral lattice $L$ with two hyperbolic planes (in particular for $L_{t}$ ) the $\widehat{\mathrm{SO}}(L)$-orbit of any $l \in L$ depends only on the norm of $l$ and its canonical image $l^{*}:=l / \operatorname{div}(l)$ in the discriminant group $\widehat{L} / L$, where the divisor 
$\operatorname{div}(l) \in \mathbb{N}$ of $l$ is the positive generator of the ideal $\left\{(x, l)_{L} \mid x \in L\right\}$. As a corollary we have

Lemma 3.2. Let $\ell_{1}, \ell_{2} \in \widehat{L}_{t}$ be two primitive vectors with the same image in the discriminant group (i.e. $\ell_{1}-\ell_{2} \in L_{t}$ ). If $\ell_{1}^{2}=\ell_{2}^{2}$, then $H_{\ell_{1}}=H_{\ell_{2}}$.

Proof. If $\ell_{1}-\ell_{2} \in L_{t}$, then $\operatorname{div}\left(2 t \ell_{1}\right)=\operatorname{div}\left(2 t \ell_{2}\right)$ and $\widehat{\mathrm{SO}}^{+}\left(L_{t}\right) \ell_{1}=\widehat{\mathrm{SO}}^{+}\left(L_{t}\right) \ell_{2}$.

Definition. Let $\ell$ be a primitive vector of $\widehat{L}_{t}$. The integer $\Delta(\ell)=2 t \ell^{2}$ is called the discriminant of $H_{\ell}$.

From the isomorphism $\widehat{L}_{t} / L_{t} \cong \mathbb{Z} / 2 t \mathbb{Z}$ one gets

Corollary $3 \cdot 3$. The number of surfaces $H_{\ell}$ with fixed discriminant $\Delta=2 t \ell^{2}$, which are not $\Gamma_{t}$-equivalent, is equal to the number of solutions

$$
\#\left\{b \bmod 2 t \mid b^{2} \equiv \Delta \bmod 4 t\right\} \text {. }
$$

The standard definition of the Humbert surfaces (see $[\mathbf{v d G}],[\mathbf{F}]$ ) is given in terms of the moduli space of abelian surfaces with polarization $(1, t)$. Let us compare both definitions.

According to (1.8) and Proposition 1.2 we may rewrite the definition of $\mathscr{H}_{\ell}$ with $\ell=(e, a,-b / 2 t, c, f) \in \widehat{L}_{t}((e, a, b, c, f)=1)$ in coordinates $\left(\tau_{i}\right)$ of $\mathbb{H}_{2}$ :

$$
\mathscr{H}_{x}^{\prime}=\psi_{t}^{-1}\left(\mathscr{H}_{\ell}\right)=\left\{\left(\begin{array}{cc}
\tau_{1} & \tau_{2} \\
\tau_{2} & \tau_{3}
\end{array}\right) \in \mathbb{H}_{2} \mid\left(\tau_{2}^{2}-\tau_{1} \tau_{3}\right) f+c \tau_{3}+b \tau_{2}+t a \tau_{1}+t e=0\right\},
$$

where $x=(t e, t a, b, c, f)$. The number

$$
2 t \ell^{2}=b^{2}-4 f(t e)-4 c(t a)=\Delta\left(\mathscr{H}_{x}^{\prime}\right) .
$$

is by definition the discriminant of $\mathscr{H}_{x}^{\prime}$. Let us introduce a lattice

$$
N_{t}=\left\{(e, a, b, c, f) \in \mathbb{Z}^{5} \mid e, a \equiv 0 \bmod t\right\} .
$$

In accordance with Proposition 1.2 and Lemma $3 \cdot 1$ we have the following decomposition of the usual (in sense of $[\mathbf{v d G}],[\mathbf{F}]$ ) Humbert surface $H_{\Delta} \subset \mathscr{A}_{t}=\Gamma_{t} \backslash \mathbb{H}_{2}$ :

$$
H_{\Delta}=\pi_{t}\left(\bigcup_{\substack{x \in N_{t}, \text { primitive } \\ \Delta(x)=\Delta}} \mathscr{H}_{x}^{\prime}\right) \cong \pi\left(\bigcup_{2 t \ell^{2}=\Delta} \underset{g \in \widehat{S O}^{+}\left(L_{t}\right)}{\bigcup_{g}} \mathscr{H}_{g \ell}\right),
$$

where one takes the summation over representatives $\ell$ from the distinct orbits and $\pi_{t}$ is the natural projection $\pi_{t}: \mathbb{H}_{2} \rightarrow \mathscr{A}_{t}$. Thus the surface $\mathscr{H}_{\ell}$ defined above corresponds to an irreducible component of the surface $H_{\Delta}$. Corollary 3.3 tells us that the number of the irreducible components of the $H_{\Delta}$ is equal to

$$
\#\left\{b \bmod 2 t \mid b^{2} \equiv \Delta \bmod 4 t\right\} .
$$

This gives a new proof of Theorem $2 \cdot 4$ in [vdG] (see p. 212).

In Section 1 we fixed a basis of the lattice $L_{t}$ such that

$$
L_{t}=U(-1) \oplus U(-1) \oplus<2 t>,
$$

where $U(-1)$ is the integral hyperbolic plane with the quadratic form $\left(\begin{array}{cc}0 & -1 \\ -1 & 0\end{array}\right)$ 
and $\langle 2 t\rangle$ is the one dimensional $\mathbb{Z}$-lattice with even quadratic form $2 t$. By $L_{t}^{(3)}$ we denote the orthogonal component in $L_{t}$ of the first hyperbolic plane

$$
L_{t}^{(3)}=\left(e_{2} \wedge e_{3}, e_{1} \wedge e_{3}-t e_{2} \wedge e_{4}, e_{4} \wedge e_{1}\right) \mathbb{Z}^{3} \subset L_{t} .
$$

It is easy to see that in any orbit $\widehat{\mathrm{SO}}\left(L_{t}\right) \ell$ there is a vector from $L_{t}^{(3)}$ and $\widehat{\mathrm{SO}}^{+}\left(L_{t}\right) \ell=\widehat{\mathrm{SO}}\left(L_{t}\right) \ell$. Thus any Humbert surface can be given in the form

$$
\mathscr{H}_{\ell}=\left\{a z_{1}+b z_{2}+c z_{3}=0\right\} \subset \mathbb{P H}_{t}^{+}, \quad \Delta\left(\mathscr{H}_{\ell}\right)=2 t \ell^{2}
$$

or

$$
\mathscr{H}_{x}^{\prime}=\left\{t a \tau_{1}+b \tau_{2}+c \tau_{3}=0\right\} \subset \mathbb{H}_{2}, \quad \Delta\left(\mathscr{H}_{x}^{\prime}\right)=b^{2}-4 t a c=2 t \ell^{2}
$$

where $\ell={ }^{t}(0, a,-b / 2 t, c, 0) \in \widehat{L}_{t}$ and $x=(t a, b, c) \in N_{t}$.

For any $d \| t$ we define the following subgroup of $\Gamma_{t}^{*}$ and the corresponding quotient space of the moduli space $\mathscr{A}_{t}$

$$
\Gamma_{t}^{(d)}=\Gamma_{t} \cup \Gamma_{t} V_{d}, \quad \mathscr{A}_{t}^{(d)}=\Gamma_{t}^{(d)} \backslash \mathbb{H}_{2} .
$$

The ramification locus of the map $\mathscr{A}_{t} \rightarrow \mathscr{A}_{t}^{(d)}$ can consist of components of different dimension. In the next theorem we describe its divisorial part $D_{t}^{(d)}$.

Theorem 3.4. Let $t$ be square free, $d>1$ and $t_{d}=t / d$. Then

$$
D_{t}^{(d)}= \begin{cases}H_{4 d} \cup H_{d} & \text { if } \quad\left(\frac{d}{4 t_{d}}\right)=1 \\ H_{4 d} & \text { if } \quad\left(\frac{d}{4 t_{d}}\right) \neq 1 \text { and }\left(\frac{d}{t_{d}}\right)=1,\end{cases}
$$

where $(a / b)$ is the generalized Kronecker symbol of the quadratic residue.

Remarks. 1. For $d=t$

$$
D_{t}^{(t)}= \begin{cases}H_{4 t} \cup H_{t} & \text { if } t \equiv 1 \bmod 4 \\ H_{4 t} & \text { otherwise. }\end{cases}
$$

In particular $D_{t}^{(t)}$ is irreducible if $t \equiv 2$ or $3 \bmod 4$ (see Corollary $\left.3 \cdot 3\right)$.

2. For $d=1$ Theorem $3 \cdot 4$ is still true if we denote by $D_{t}^{(1)}$ the divisorial part of the branch locus of the covering $\mathbb{H}_{2} \rightarrow \mathscr{A}_{t}$. We note that $D_{t}^{(1)}$ was found in $[\mathbf{B}]$ by another method.

Corollary 3.5. Let $t$ be square free. The divisorial part $D_{t}^{*}$ of the ramification locus of the map $\mathscr{A}_{t} \rightarrow \mathscr{A}_{t}^{*}$, where $\mathscr{A}_{t}^{*}=\Gamma_{t}^{*} \backslash \mathbb{H}_{2}$ is the 'minimal' Siegel modular threefold corresponding to polarization of type $(1, t)$, is the union of the following Humbert surfaces

$$
D_{t}^{*}=\bigcup_{d \mid t}\left(\varepsilon_{1}(d) H_{4 d} \cup \varepsilon_{2}(d) H_{d}\right)
$$

where $\varepsilon_{1}(d)=1$ if $\left(d / t_{d}\right)=1, \varepsilon_{2}(d)=1$ if $d$ is odd and $\left(d / 4 t_{d}\right)=1$ and they equal 0 in all other cases. Moreover none of the above Humbert surfaces is $\Gamma_{t}$-equivalent.

Proof of corollary We have to prove only the last statement. If $\ell_{1}$ and $\ell_{2} \in \widehat{L}_{t}$ are two primitive vectors with norms $\ell_{1}^{2}=2 / t_{d_{1}}, \ell_{2}^{2}=1 /\left(2 t_{d_{2}}\right)$, then $\ell_{1}^{2} \neq \ell_{2}^{2}$, since $t$ is square free. 
We break up the proof of Theorem $3 \cdot 4$ into several lemmas.

Let us consider a reflection with respect to a vector $v \in L_{t} \otimes \mathbb{R}$ :

$$
\sigma_{v}(x)=x-\frac{2(x, v)}{(v, v)} v .
$$

It is known that $\sigma_{v} \in \mathrm{O}_{\mathbb{R}}^{+}\left(L_{t}\right)$ if and only if $v^{2}>0$. (This follows from the definition of the real spin norm.) If $\sigma_{v} \in \mathrm{O}_{\mathbb{R}}^{+}\left(L_{t}\right)$, then the set Fix $\mathbb{P H}_{t}^{+}\left(\sigma_{v}\right)$ of fix points of $\sigma_{v}$ on $\mathbb{P H}_{t}^{+}$is a complex surface $\mathscr{H}_{v}$. The opposite statement is also true.

Lemma 3.6. Let us suppose that the set of fix points of $\sigma \in \mathrm{SO}_{\mathbb{R}}^{+}\left(L_{t}\right)$ on $\mathbb{P H}_{t}^{+}$is a complex surface. Then $-\sigma$ is a reflection with respect to a vector $v \in L_{t} \otimes \mathbb{R}$.

Proof. Over $\mathbb{R}$ one can reduce the quadratic form $S_{t}$ to $S=\operatorname{diag}\left(E_{3},-E_{2}\right)$. The maximal compact subgroup $K_{\mathbb{R}}$ of the orthogonal group $\mathrm{SO}_{\mathbb{R}}^{+}(S)$ is isomorphic to $\mathrm{SO}(3) \times \mathrm{SO}(2)$ consisting of all elements which fix the point $Z_{\mathbf{i}}={ }^{t}(0,0,0, i, 1) \in \mathbb{P H}_{t}^{+}$. Since the group $\mathrm{SO}_{\mathbb{R}}^{+}(S)$ acts transitively on the homogeneous domain we can suppose that $\sigma=\operatorname{diag}(A, B) \in K$ where $A \in \mathrm{SO}(3)$ and $B \in \mathrm{SO}(2)$. If $B \neq \pm E_{2}$, then $B$ has only one fix point $\mathbf{i}={ }^{t}(i, 1)$ on the projective line. If $\sigma=\operatorname{diag}(A, B)$ has at least three fixed points, then $B$ has an eigenvalue $\lambda$ of order two. $A$ and $B$ are orthogonal, thus all eigenvalues of $\sigma$ are equal to \pm 1 .

There are two possibilities for the set of eigenvalues of $\sigma$

$$
\{\lambda(\sigma)\}=\{1,-1,-1,-1,-1\} \quad \text { or } \quad\{1,1,1,-1,-1\} .
$$

In the first case $-\sigma$ is a reflection. In the second case $\sigma$ can be written as a product of two reflections $\sigma_{v} \sigma_{u}$ with orthogonal $u$ and $v$. Thus $\operatorname{Fix}_{\mathbb{P H}_{t}^{+}}(\sigma)=\mathscr{H}_{u} \cap \mathscr{H}_{v}$ and we have proved the lemma for non-trivial $B$.

If $B= \pm E_{2}$, then the same arguments show that $\sigma$ is conjugate to

$$
D=\left(\begin{array}{ccc}
B_{1} & 0 & 0 \\
0 & \pm 1 & 0 \\
0 & 0 & \pm E_{2}
\end{array}\right) \quad B_{1} \in \mathrm{SO}(2)
$$

if $\sigma$ has at least two fixed points. If $B_{1} \neq \pm E_{2}$, then Fix $\mathbb{P H}_{t}^{+}(D)$ is a subset of $\mathscr{H}_{x} \bigcap \mathscr{H}_{y}$, where $x$ and $y$ form an orthogonal basis of the plane of rotation of $B_{1} \in \mathrm{SO}(2)$.

Lemma 3.7. There is a one to one correspondence between the irreducible components $H$ of the divisorial part $D_{t}^{(d)}$ and the surfaces $H_{\ell}$ defined by reflections $\sigma_{\ell} \in \Gamma_{t} V_{d}$.

Proof. By $\mathscr{H}$ we denote an irreducible surface in $\mathbb{H}_{2}$ whose image is $H$. Let us suppose that $\mathscr{H}=\operatorname{Fix}_{\mathbb{H}_{2}}(G)$ with $G \in \Gamma_{t} V_{d}$. In accordance with Proposition 1.2 and Lemma $3 \cdot 6 \Psi(G)=\sigma_{\ell}$ is a reflection. Moreover $\psi_{t}(\mathscr{H})=\mathscr{H}_{\ell} \subset \mathbb{P H}_{t}^{+}$and $\sigma_{\ell}$ induces multiplication by $\xi_{d}$ on the discriminant group $A_{t}$.

The reflection

$$
\sigma_{\ell}(x)=x-\frac{2(x, \ell)}{(\ell, \ell)} \ell
$$

depends only on the line $\left\langle\ell>\right.$ defined by $\ell \in L_{t}$. It follows from the definition that $\sigma_{\ell}$ keeps the lattice $L_{t}$ invariant if and only if $\ell^{2} \mid 2 D$ where $D=\operatorname{div}(\ell)$ (see the definition before Lemma 3.2). The surface $H$ depends only on the class $\left\{\gamma G \gamma^{-1} \mid \gamma \in \Gamma_{t}\right\}$ and

$$
\Psi\left(\gamma G \gamma^{-1}\right)=\beta \sigma_{\ell} \beta^{-1}=\sigma_{\beta \ell} \quad\left(\gamma \in \Gamma_{t}, \beta=\Psi(\gamma) \in \widehat{\mathrm{SO}}^{+}\left(L_{t}\right)\right)
$$


Therefore in order to find all Humbert surfaces in the divisorial part we have to classify the $\widehat{\mathrm{SO}}\left(L_{t}\right)$-orbits of vectors $\ell \in L_{t}$ with the additional condition $\ell^{2} \mid 2 \operatorname{div}(\ell)$.

Lemma 3.8. Let $t$ be an arbitrary positive integer and $d \| t$. There is a one to one correspondence between the $\widehat{\mathrm{SO}}\left(L_{t}\right)$-conjugacy classes of reflections $\sigma_{\ell}$ in the coset $\left(-\Gamma_{t} V_{d}\right)$ and the orbits of the primitive vectors in $L_{t}$, which satisfy the following conditions:

$$
\ell^{2}=2 d \quad \text { and } \begin{cases}\operatorname{div}(\ell)=2 t_{d} & \text { if }\left(\frac{d}{4 t_{d}}\right)=1 \\ \operatorname{div}(\ell)=t_{d} & \text { if }\left(\frac{d}{t_{d}}\right)=1 .\end{cases}
$$

Proof. One can suppose that $\ell={ }^{t}(0, a, b, c, 0) \in L_{t}$ and $(a, b, c)=1$. For such $\ell$ the matrix of $\sigma_{\ell}$ has the following form

$$
\sigma_{\ell}=\left(\begin{array}{ccccc}
1 & 0 & 0 & 0 & 0 \\
0 & 1+2 c a / \ell^{2} & -4 t b a / \ell^{2} & 2 a^{2} / \ell^{2} & 0 \\
0 & 2 c b / \ell^{2} & 1-4 t b^{2} / \ell^{2} & 2 a b / \ell^{2} & 0 \\
0 & 2 c^{2} / \ell^{2} & -4 t b c / \ell^{2} & 1+2 a c / \ell^{2} & 0 \\
0 & 0 & 0 & 0 & 1
\end{array}\right),
$$

where $\ell^{2}=2 t b^{2}-2 a c .-\sigma_{\ell} \in \mathrm{SO}_{\mathbb{R}}^{+}$if and only if $\ell^{2}>0$. On the discriminant group $-\sigma_{\ell}$ defines multiplication by

$$
\xi(\ell)=\frac{4 t}{\ell^{2}} b^{2}-1 .
$$

By definition $D=\operatorname{div}(\ell)=(a, 2 t b, c)$, therefore $D \mid 2 t$ and $D\left|\ell^{2}\right| 2 D$. We put

$$
\ell={ }^{t}\left(0, D a_{1}, b, D c_{1}, 0\right) \quad \text { with }\left(a_{1}, \frac{2 t b}{D}, c_{1}\right)=1 .
$$

We have to consider four cases:

$$
\ell^{2}=D \quad \text { or } \quad \ell^{2}=2 D \text { and } D \mid t \quad \text { or } D \nmid t .
$$

(1) Let us suppose that $D \mid t\left(t_{D}=t / D\right)$. Then we have

$$
\begin{gathered}
\ell^{2}=2 D \Longleftrightarrow 1=t_{D} b^{2}-D a_{1} c_{1} \Longleftrightarrow\left(D, t_{D}\right)=1 \&\left(\frac{t_{D}}{D}\right)=1, \\
\xi\left(-\sigma_{\ell}\right)=2 t_{D} b^{2}-1=\left\{\begin{array}{rl}
-1 & \bmod 2 t_{D} \\
1 & \bmod 2 D
\end{array} \Longrightarrow-\sigma_{\ell} \in V_{t_{D}} \Gamma_{t}\right.
\end{gathered}
$$

(see $(1 \cdot 5))$. The case $\ell^{2}=D$ leads trivially to a contradiction.

(2) Let us suppose that $D \mid 2 t$, but $t / D \notin \mathbb{Z}$. In this case $D=2 D_{1}, D_{1} \mid t$ and $t_{D_{1}}$ is odd. For such $D$ we have

$$
\begin{gathered}
\ell^{2}=D \Longleftrightarrow 1=t_{D_{1}} b^{2}-4 D_{1} a_{1} c_{1} \Longleftrightarrow\left(D_{1}, t_{D_{1}}\right)=1 \&\left(t_{D_{1}} \text { odd }\right) \&\left(\frac{t_{D_{1}}}{4 D_{1}}\right)=1, \\
\xi\left(-\sigma_{\ell}\right)=2 t_{D_{1}} b^{2}-1=\left\{\begin{array}{rl}
-1 & \bmod 2 t_{D_{1}} \\
1 & \bmod 2 D_{1}
\end{array} \Longrightarrow-\sigma_{\ell} \in V_{t_{D_{1}}} \Gamma_{t} .\right.
\end{gathered}
$$

The case $\ell^{2}=2 D$ leads to a contradiction to the primitivity of $\ell$.

For square free $t$ the system of the surfaces $\left\{H_{\ell^{*}}\right\}$, where $\ell^{*}=\ell /(\operatorname{div}(\ell))$ and 
$\ell$ satisfies the condition of Lemma $3 \cdot 8$, contains all irreducible components of the Humbert surfaces from Theorem $3 \cdot 4$. This finishes the proof of Theorem $3 \cdot 4$.

The next corollary follows immediately from the proof of Lemma $3 \cdot 8$.

Corollary 3.9. Let $t$ be square free and $d \| t$. If $\left(d / t_{d}\right)=1$ and $\left(d / 4 t_{d}\right) \neq 1$ then there $i s$, up to conjugation with respect to $\Gamma_{t}$, exactly one, and if $\left(d / 4 t_{d}\right)=1$ then there are exactly two involutions in $\Gamma_{t} V_{d}$. They are $\Psi^{-1}\left(-\sigma_{\ell_{1}}\right)$ (in the both cases), and $\Psi^{-1}\left(-\sigma_{\ell_{2}}\right)$ (in the second case), where

$$
\begin{aligned}
& \ell_{1}={ }^{t}\left(0, a_{1}, \frac{b_{1}}{t_{d}}, c_{1}, 0\right) \in \widehat{L}_{t}, \quad\left(a_{1}, b_{1}, c_{1}\right)=1, \quad d b_{1}^{2}-t_{d} a_{1} c_{1}=1 \\
& \ell_{2}={ }^{t}\left(0, a_{2}, \frac{b_{2}}{2 t_{d}}, c_{2}, 0\right) \in \widehat{L}_{t}, \quad\left(a_{2}, b_{2}, c_{2}\right)=1, \quad d b_{2}^{2}-4 t_{d} a_{2} c_{2}=1 .
\end{aligned}
$$

Remarks. 1. It is possible to apply Lemma $3 \cdot 8$, which has been proved for any integer $t$, to classify the divisorial part of the ramification locus of the covering $\mathscr{A}_{t} \rightarrow \mathscr{A}_{t}^{*}$ for any integer $t$.

2. Using the same method one can construct divisors on a homogeneous domain of type IV of any dimension $n$.

The ramification locus can also have components of smaller dimension. The proof of Lemma $3 \cdot 6$ shows us that the orthogonal group can contain a rotation in the positive definite subplane of the lattice $L_{t}$.

Example (Brasch). The following example is due to Brasch. It shows that in general the ramification locus of the map $\mathscr{A}_{t} \rightarrow \mathscr{A}_{t}^{*}$ contains other components apart from the divisorial part described above. Let $t \equiv 1 \bmod 4$. For an integer $f>0$ put

$$
c=-f^{2} t-1, \quad g=f^{2} .
$$

Then the matrix

$$
N=\left(\begin{array}{cccc}
-f \sqrt{ } t & 1 / \sqrt{ } t & 0 & f \sqrt{ } t \\
c \sqrt{ } t & 0 & f \sqrt{ } t & f^{2} t \sqrt{ } t \\
c \sqrt{ } t & 0 & f \sqrt{ } t & -c \sqrt{ } t \\
0 & 1 / \sqrt{ } t & -1 / \sqrt{ } t & 0
\end{array}\right)
$$

is an element of $\Gamma_{t}^{*}$. One immediately checks that $N^{2}=-E_{4}$. The fixed point set Fix $N$ is a curve (ef. [B, Hilfssatz $2 \cdot 5 \cdot 3]$ ). Moreover the curve Fix $N$ is not contained in the fixed point set Fix $I$ of an involution $I$ in $\Gamma_{t}^{*}$. This would namely imply that $I N=-N I$. Now using the explicit form for $N$ which follows from [B, Hilfssatz 2.8], a lengthy but straightforward calculation shows $c=-1$, a contradiction.

As a next step we want to interpret the surfaces $H_{t}$, resp. $H_{4 t}$ as moduli spaces of abelian surfaces with real multiplication. It is well known that there is a close connection between Hilbert modular surfaces and Humbert surfaces [F], [vdG, chapter IX]. Here we want to determine precisely which Hilbert modular surfaces correspond to $H_{t}$, resp. $H_{4 t}$. Consider the ring $\mathfrak{o}$ of integers in the number field $\mathbb{Q}(\sqrt{ } t)$ and recall that

$$
\mathfrak{v}=\mathbb{Z}+\mathbb{Z} \omega, \quad \omega=\frac{1}{2}(1+\sqrt{ } t) \quad \text { if } t \equiv 1 \bmod 4
$$


resp.

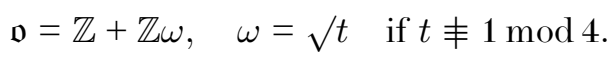

The Hilbert modular group $\mathrm{SL}_{2}(\mathfrak{p})$ acts on $\mathbb{H}_{1} \times \mathbb{H}_{1}$ by

$$
\left(\begin{array}{ll}
\alpha & \beta \\
\gamma & \delta
\end{array}\right)\left(z_{1}, z_{2}\right)=\left(\frac{\alpha z_{1}+\beta}{\gamma z_{1}+\delta}, \frac{\alpha^{\prime} z_{2}+\beta^{\prime}}{\gamma^{\prime} z_{2}+\delta^{\prime}}\right)
$$

where ${ }^{\prime}$ denotes the Galois automorphism $\sqrt{ } t \mapsto-\sqrt{ } t$. The quotient space $Y=$ $\mathrm{SL}_{2}(\mathfrak{v}) \backslash \mathbb{H}_{1} \times \mathbb{H}_{1}$ is the standard Hilbert modular surface associated to $\mathbb{Q}(\sqrt{ } t)$. Let $\sigma$ be the involution which interchanges the two factors of $\mathbb{H}_{1} \times \mathbb{H}_{1}$, i.e.

$$
\sigma\left(z_{1}, z_{2}\right)=\left(z_{2}, z_{1}\right)
$$

Then the symmetric Hilbert modular group is

$$
\mathrm{SL}_{2}^{\sigma}(\mathfrak{p})=\mathrm{SL}_{2}(\mathfrak{p}) \cup \sigma \mathrm{SL}_{2}(\mathfrak{p})
$$

and $Y^{\sigma}=\mathrm{SL}_{2}^{\sigma}(\mathfrak{D}) \backslash \mathbb{H}_{1} \times \mathbb{H}_{1}$ is the corresponding symmetric Hilbert modular surface. We shall first consider the Humbert surface $H_{t}$. In particular we assume that $t \equiv 1$ $\bmod 4$. To every point $\left(z_{1}, z_{2}\right) \in \mathbb{H}_{1} \times \mathbb{H}_{1}$ one can associate the lattice

$$
\Lambda_{\left(z_{1}, z_{2}\right)}=\mathfrak{v}\left(\begin{array}{c}
z_{1} \\
z_{2}
\end{array}\right)+\mathfrak{o}\left(\begin{array}{l}
1 \\
1
\end{array}\right)=\mathbb{Z}\left(\begin{array}{c}
z_{1} \\
z_{2}
\end{array}\right)+\mathbb{Z}\left(\begin{array}{c}
\eta z_{1} \\
\eta^{\prime} z_{2}
\end{array}\right)+\mathbb{Z}\left(\begin{array}{c}
-\eta^{\prime} / \sqrt{ } t \\
\eta / \sqrt{ } t
\end{array}\right)+\mathbb{Z}\left(\begin{array}{c}
\sqrt{ } t \\
-\sqrt{ } t
\end{array}\right)
$$

where $\eta=\sqrt{ }(t) \omega$. The form

$$
E\left(\left(x_{1}, x_{2}\right),\left(y_{1}, y_{2}\right)\right)=\operatorname{Im}\left(\frac{x_{1} \bar{y}_{1}}{\operatorname{Im} z_{1}}+\frac{x_{2} \bar{y}_{2}}{\operatorname{Im} z_{2}}\right)
$$

defines a Riemann form for $\Lambda_{t}$. With respect to the basis given above this is just the alternating form $W_{t}$. The torus

$$
A_{\left(z_{1}, z_{2}\right)}=\mathbb{C}^{2} / \Lambda_{\left(z_{1}, z_{2}\right)}
$$

is hence a $(1, t)$-polarized abelian surface with real multiplication in $\mathfrak{o}$. The Hilbert modular surface $Y$ is the moduli space of these objects. The $2: 1$ cover $Y \rightarrow Y^{\sigma}$ identifies abelian surfaces whose real multiplication differs by the Galois conjugation. We have a 'forgetful' map

$$
\Phi: Y^{\sigma} \rightarrow \mathscr{A}_{t}
$$

Theorem 3·10. Assume $t \equiv 1 \bmod$. The Humbert surface $H_{t}$ is the image of the symmetric Hilbert modular surface $Y^{\sigma}$ under the natural morphism $\Phi: Y^{\sigma} \rightarrow \mathscr{A}_{t}$ which is of degree 1 onto its image.

Before giving the proof we turn to the Humbert surfaces $H_{4 t}$. Consider the ring

$$
\mathfrak{v}_{2}=\mathbb{Z}+\mathbb{Z} \sqrt{ } t
$$

Note that this is an order in $\mathfrak{v}$ if $t \equiv 1 \bmod 4$, whereas $\mathfrak{v}=\mathfrak{o}_{2}$ if $t \neq 1 \bmod 4$. Let

$$
\tilde{\mathfrak{o}}_{2}=\frac{1}{2} \mathbb{Z}+\frac{1}{2} \sqrt{ } t \mathbb{Z}, \quad \tilde{\mathfrak{o}}_{2}^{-1}=2 \mathbb{Z}+2 \sqrt{ } t \mathbb{Z} .
$$

The group

$$
\mathrm{SL}_{2}\left(\mathfrak{o}_{2}, \tilde{\mathfrak{o}}_{2}\right)=\left\{\left(\begin{array}{ll}
a & b \\
c & d
\end{array}\right) \mid a, d \in \mathfrak{o}_{2}, b \in \tilde{\mathfrak{o}}_{2}, c \in \tilde{\mathfrak{o}}_{2}^{-1}, a d-b c=1\right\}
$$


acts on $\mathbb{H}_{1} \times \mathbb{H}_{1}$ as well as its symmetric counterpart

$$
\mathrm{SL}_{2}^{\sigma}\left(\mathfrak{o}_{2}, \tilde{\mathfrak{o}}_{2}\right)=\mathrm{SL}_{2}\left(\mathfrak{o}_{2}, \tilde{\mathfrak{o}}_{2}\right) \cup \sigma \mathrm{SL}_{2}\left(\mathfrak{o}_{2}, \tilde{\mathfrak{o}}_{2}\right)
$$

Let

$$
\tilde{Y}=S L_{2}\left(\mathfrak{o}_{2}, \tilde{\mathfrak{o}}_{2}\right) \backslash \mathbb{H}_{1} \times \mathbb{H}_{1}, \quad \tilde{Y}^{\sigma}=\mathrm{SL}_{2}^{\sigma}\left(\mathfrak{o}_{2}, \tilde{\mathfrak{o}}_{2}\right) \backslash \mathbb{H}_{1} \times \mathbb{H}_{1}
$$

be the corresponding Hilbert modular surfaces. Again the Riemann form $E$ induces a $(1, t)$-polarization on the tori

$$
A_{\left(z_{1}, z_{2}\right)}=\mathbb{C}^{2} / \Lambda_{\left(z_{1}, z_{2}\right)}
$$

where for $t \equiv 1 \bmod 4$

$$
\Lambda_{\left(z_{1}, z_{2}\right)}=\mathbb{Z}\left(\begin{array}{c}
z_{1} \\
z_{2}
\end{array}\right)+\mathbb{Z}\left(\begin{array}{c}
2 \eta z_{1} \\
2 \eta^{\prime} z_{2}
\end{array}\right)+\mathbb{Z}\left(\begin{array}{c}
-\eta^{\prime} / \sqrt{ } t \\
\eta / \sqrt{ } t
\end{array}\right)+\mathbb{Z}\left(\begin{array}{c}
\sqrt{ } t / 2 \\
-\sqrt{ } t / 2
\end{array}\right)
$$

resp. $t$ 丰 $1 \bmod 4$

$$
\Lambda_{\left(z_{1}, z_{2}\right)}=\mathbb{Z}\left(\begin{array}{c}
z_{1} \\
z_{2}
\end{array}\right)+\mathbb{Z}\left(\begin{array}{c}
\omega z_{1} \\
\omega^{\prime} z_{2}
\end{array}\right)+\mathbb{Z}\left(\begin{array}{c}
1 / 2 \\
1 / 2
\end{array}\right)+\mathbb{Z}\left(\begin{array}{c}
\omega / 2 \\
\omega^{\prime} / 2
\end{array}\right)
$$

Hence $\tilde{Y}$, resp. $\widetilde{Y}^{\sigma}$ are moduli spaces of $(1, t)$-polarized abelian surfaces with real multiplication in $\mathfrak{b}_{2}$ and as before we have a canonical map

$$
\widetilde{\Phi}: \widetilde{Y}^{\sigma} \rightarrow \mathscr{A}_{t} \text {. }
$$

TheOREM 3.11. The Humbert surface $H_{4 t}$ is the image of the symmetric Hilbert modular surface $\widetilde{Y}^{\sigma}$ under the natural map $\widetilde{\Phi}: \widetilde{Y}^{\sigma} \rightarrow \mathscr{A}_{t}$ which is of degree 1 onto its image.

Proof of Theorems $3 \cdot 10$ and $3 \cdot 11$. We shall treat the case of $H_{4 t}$ and $t \equiv 1 \bmod 4$ in detail and then comment on the other cases. The proof is similar to the proofs in [HL, section 0], cf. also [F, Abschnitt 3]. Let

$$
R=\left(\begin{array}{cc}
1 & 2 \eta \\
1 & 2 \eta^{\prime}
\end{array}\right)
$$

and consider the map

$$
\begin{aligned}
\widehat{\Phi}: \mathbb{H}_{1} \times \mathbb{H}_{1} & \rightarrow \mathbb{H}_{2} \\
\left(z_{1}, z_{2}\right) & \mapsto{ }^{t} R\left(\begin{array}{cc}
z_{1} & 0 \\
0 & z_{2}
\end{array}\right) R .
\end{aligned}
$$

Then

$$
\operatorname{Im} \widehat{\Phi}=\left\{-\left(t^{2}-t\right) \tau_{1}+2 t \tau_{2}-\tau_{3}=0\right\}=\mathscr{H}_{4 t}^{\prime}
$$

and modulo

$$
X=\left(\begin{array}{cccc}
1 & 0 & 0 & 0 \\
t & 1 & 0 & 0 \\
0 & 0 & 1 & -t \\
0 & 0 & 0 & 1
\end{array}\right) \in \Gamma_{t}
$$

this is equivalent to $\mathscr{H}_{4 t}=\left\{t \tau_{1}-\tau_{3}=0\right\}$. Let $A_{\widehat{\Phi}\left(z_{1}, z_{2}\right)}$ be the abelian surface associ- 
ated to the period matrix

$$
\left({ }^{t} R\left(\begin{array}{cc}
z_{1} & 0 \\
0 & z_{2}
\end{array}\right) R\left(\begin{array}{cc}
1 & 0 \\
0 & t
\end{array}\right)\right)
$$

Then $A_{\left(z_{1}, z_{2}\right)}$ and $A_{\widehat{\Phi}\left(z_{1}, z_{2}\right)}$ are isomorphic as polarized abelian surfaces since

$$
{ }^{t} R\left(\begin{array}{cccc}
z_{1} & 2 \eta z_{1} & -\eta^{\prime} / \sqrt{ } t & \sqrt{ } t / 2 \\
z_{2} & 2 \eta^{\prime} z_{2} & \eta / \sqrt{ } t & -\sqrt{ } t / 2
\end{array}\right)=\left({ }^{t} R\left(\begin{array}{cc}
z_{1} & 0 \\
0 & z_{2}
\end{array}\right) R\left(\begin{array}{cc}
1 & 0 \\
0 & t
\end{array}\right)\right) .
$$

Hence $\widehat{\Phi}$ is a lift of the map $\widetilde{\Phi}$. Next we consider the homomorphism

$$
\begin{gathered}
\Psi: \mathrm{SL}_{2}(\mathbb{R}) \times \mathrm{SL}_{2}(\mathbb{R}) \longrightarrow \mathrm{Sp}_{4}(\mathbb{R}) \\
\Psi\left(\left(\begin{array}{ll}
a_{1} & b_{1} \\
c_{1} & d_{1}
\end{array}\right),\left(\begin{array}{cc}
a_{2} & b_{2} \\
c_{2} & d_{2}
\end{array}\right)\right)=\left(\begin{array}{cc}
{ }^{t} R & 0 \\
0 & R^{-1}
\end{array}\right)\left(\begin{array}{ll}
d\left(a_{1}, a_{2}\right) & d\left(b_{1}, b_{2}\right) \\
d\left(c_{1}, c_{2}\right) & d\left(d_{1}, d_{2}\right)
\end{array}\right)\left(\begin{array}{cc}
{ }^{t} R & 0 \\
0 & R
\end{array}\right)
\end{gathered}
$$

where $d\left(a_{1}, a_{2}\right)=\left(\begin{array}{cc}a_{1} & 0 \\ 0 & a_{2}\end{array}\right)$, etc. Via the embedding

$$
\begin{aligned}
\mathrm{SL}_{2}(\mathbb{Q}(\sqrt{ } t)) & \rightarrow \mathrm{SL}_{2}(\mathbb{R}) \times \mathrm{SL}_{2}(\mathbb{R}) \\
\left(\begin{array}{ll}
\alpha & \beta \\
\gamma & \delta
\end{array}\right) & \mapsto\left(\left(\begin{array}{ll}
\alpha & \beta \\
\gamma & \delta
\end{array}\right),\left(\begin{array}{ll}
\alpha^{\prime} & \beta^{\prime} \\
\gamma^{\prime} & \delta^{\prime}
\end{array}\right)\right)
\end{aligned}
$$

this also defines a homomorphism

$$
\widehat{\Psi}: \mathrm{SL}_{2}(\mathbb{Q}(\sqrt{ } t)) \rightarrow \mathrm{Sp}_{4}(\mathbb{R}) .
$$

For $J=\left(\begin{array}{ll}0 & 1 \\ 1 & 0\end{array}\right)$ we find that

$$
S=\left(\begin{array}{cc}
{ }^{t} R J^{t} R^{-1} & 0 \\
0 & R^{-1} J R
\end{array}\right) \in \Gamma_{t} .
$$

Clearly $S$ is an involution. Setting $\widehat{\Psi}(\sigma)=S$ we can extend $\widehat{\Psi}$ to a homomorphism

$$
\widehat{\Psi}_{\sigma}: \mathrm{SL}_{2}^{\sigma}(\mathbb{Q}(\sqrt{ } t)) \rightarrow \mathrm{Sp}_{4}(\mathbb{R})
$$

and one checks easily that $\widehat{\Phi}$ is $\widehat{\Psi}_{\sigma}$-equivariant. Let $G$, resp. $G_{\mathbb{R}}$ be the stabilizer of $\mathscr{H}_{4 t}^{\prime}$ in $\Gamma_{t}$, resp. $\mathrm{Sp}_{4}(\mathbb{R})$. As in [HL, lemma 0.8], cf. also [F, Korollar 3.2.8] one shows that $G_{\mathbb{R}}$ is the group generated by the image of $\Psi$ and $S$. The result follows if we can prove that $G=\widehat{\Psi}_{\sigma}\left(\mathrm{SL}_{2}^{\sigma}\left(\mathfrak{o}_{2}, \tilde{\mathfrak{o}}_{2}\right)\right)$. For this it is now enough to prove the following.

Lemma 3-12. Let $\left(\begin{array}{ll}a_{i} & b_{i} \\ c_{i} & d_{i}\end{array}\right) \in \mathrm{SL}_{2}(\mathbb{R}) ; i=1,2$ and assume that

$$
M=\left(\begin{array}{cc}
{ }^{t} R & 0 \\
0 & R^{-1}
\end{array}\right)\left(\begin{array}{ll}
d\left(a_{1}, a_{2}\right) & d\left(b_{1}, b_{2}\right) \\
d\left(c_{1}, c_{2}\right) & d\left(d_{1}, d_{2}\right)
\end{array}\right)\left(\begin{array}{cc}
{ }^{t} R^{-1} & 0 \\
0 & R
\end{array}\right) \in \Gamma_{t} .
$$

Then $a_{1}, d_{1} \in \mathfrak{o}_{2}, b_{1} \in \tilde{\mathfrak{o}}_{2}, c_{1} \in \tilde{\mathfrak{o}}_{2}^{-1}$ and $a_{2}=a_{1}^{\prime}, b_{2}=b_{1}^{\prime}, c_{2}=c_{1}^{\prime}, d_{2}=d_{2}^{\prime}$.

Proof of the lemma. We write $M=\left(\begin{array}{cc}A & B \\ C & D\end{array}\right)$. Straightforward calculation gives

$$
A=\frac{1}{2\left(\eta^{\prime}-\eta\right)}\left(\begin{array}{cc}
2 \eta^{\prime} a_{1}-2 \eta a_{2} & -a_{1}+a_{2} \\
4 \eta \eta^{\prime}\left(a_{1}-a_{2}\right) & -2 \eta a_{1}+2 \eta^{\prime} a_{2}
\end{array}\right) \text {. }
$$


From $A_{12} \in \mathbb{Z}$ we find $\left(a_{1}-a_{2}\right) /(2 \sqrt{ } t) \in \mathbb{Z}$, i.e.

$$
a_{2}=a_{1}+2 n \sqrt{ } t, \quad n \in \mathbb{Z} .
$$

$A_{11} \in \mathbb{Z}$ gives $a_{1} \omega^{\prime}+a_{2} \omega \in \mathbb{Z}$. Hence

$$
a_{1}+2 n \sqrt{ } t \omega^{\prime}=a_{1}\left(\omega+\omega^{\prime}\right)+2 n \sqrt{ } t \omega^{\prime}=a_{1} \omega+a_{2} \omega^{\prime} \in \mathbb{Z} .
$$

I.e. $a_{1} \in \mathbb{Z}+\mathbb{Z} \sqrt{ } t=\mathfrak{o}_{2}$. We can write $a_{1}=\alpha+\beta \sqrt{ } t$ with $\alpha, \beta \in \mathbb{Z}$. By $3 \cdot 2$ we have $a_{1}+n(\sqrt{ } t+t) \in \mathbb{Z}$ and hence $\beta=-n$. But then $a_{2}=a_{1}-2 \beta \sqrt{ } t=\alpha-\beta \sqrt{ } t=a_{1}^{\prime}$. Note also that for $a_{1} \in \mathfrak{o}_{2}$ and $a_{2}=a_{1}^{\prime}$ one has $A \in\left(\begin{array}{cc}\mathbb{Z} & \mathbb{Z} \\ t \mathbb{Z} & \mathbb{Z}\end{array}\right)$. Similarly we obtain

$$
B=\left(\begin{array}{cc}
b_{1}+b_{2} & 2 \eta b_{1}+2 \eta^{\prime} b_{2} \\
2 \eta b_{1}+2 \eta^{\prime} b_{2} & 4 \eta^{2} b_{1}+4 \eta^{\prime 2} b_{2}
\end{array}\right) .
$$

Since $B_{11} \in \mathbb{Z}$ we find that $b_{2}=n-b_{1}$ for some $n \in \mathbb{Z}$. Using this and $B_{12} \in t \mathbb{Z}$ one concludes that

$$
\left(b_{1} \omega-b_{2} \omega^{\prime}\right) \in \frac{\sqrt{ } t}{2} \mathbb{Z}
$$

Hence

$$
b_{1}-n \omega^{\prime}=b_{1}\left(\omega+\omega^{\prime}\right)-n \omega^{\prime}=b_{1} \omega-b_{2} \omega^{\prime} \in \frac{\sqrt{ } t}{2} \mathbb{Z} .
$$

This shows $b_{1}, b_{2} \in \tilde{\mathfrak{o}}_{2}$. Writing $b_{1}=(\alpha+\beta \sqrt{ } t) / 2$ with $\alpha, \beta \in \mathbb{Z}$ one has from (3.3)

$$
\frac{\alpha}{2}+\frac{\beta}{2} \sqrt{ } t-n \omega^{\prime} \in \frac{\sqrt{ } t}{2} \mathbb{Z}
$$

i.e. $\alpha=n$. Hence $b_{2}=n-b_{1}=(\alpha-\beta \sqrt{ } t) / 2=b_{1}^{\prime}$. Conversely if $b_{1} \in \tilde{\mathfrak{o}}_{2}$ and $b_{2}=b_{1}^{\prime}$, then $B \in\left(\begin{array}{cc}\mathbb{Z} & t \mathbb{Z} \\ t \mathbb{Z} & t \mathbb{Z}\end{array}\right)$. For $C$ one computes

$$
C=\frac{1}{4 t}\left(\begin{array}{cc}
4 \eta^{\prime 2} c_{1}+4 \eta^{2} c_{2} & -2 \eta^{\prime} c_{1}-2 \eta c_{2} \\
-2 \eta^{\prime} c_{1}-2 \eta c_{2} & c_{1}+c_{2}
\end{array}\right)
$$

Comparing this with the situation for $\mathrm{B}$ one finds that $c_{1} \in \tilde{\mathfrak{o}}_{2}^{-1}, c_{2}=c_{1}^{\prime}$. Finally

$$
D=\frac{1}{2\left(\eta^{\prime}-\eta\right)}\left(\begin{array}{cc}
2 \eta^{\prime} d_{1}-2 \eta d_{2} & 4 \eta \eta^{\prime}\left(d_{1}-d_{2}\right) \\
-d_{1}+d_{2} & -2 \eta d_{1}+2 \eta^{\prime} d_{2}
\end{array}\right) .
$$

This case is analogous to $\mathrm{A}$ and one obtains $d_{1} \in \mathfrak{o}_{2}, d_{2}=d_{1}^{\prime}$. This proves Lemma $3 \cdot 12$.

The case of the Humbert surface $H_{t}, t \equiv 1 \bmod 4$ can be treated in the same manner if we choose $R=\left(\begin{array}{cc}1 & \eta \\ 1 & \eta^{\prime}\end{array}\right)$. This is analogous to [HL, section 0$]$ where the case $t=5$ was done. Finally we have to deal with $H_{4 t}$ in case $t \neq 1 \bmod 4$. Here we can choose $R=\left(\begin{array}{cc}1 & \omega \\ 1 & \omega^{\prime}\end{array}\right), \omega=\sqrt{ } t$. In this case

$$
\operatorname{Im} \widehat{\Phi}=\left\{t \tau_{1}-\tau_{3}=0\right\}=\mathscr{H}_{4 t}
$$

and the above arguments go through essentially unchanged. 


\section{REFERENCES}

[Al] N. D. Allan. The problem of the maximality of arithmetic groups. In Algebraic groups and discontinuous subgroups. Proc. of Sympos. Pure Math., vol. IX (Amer. Math. Soc., 1966), 104-109

[BPV] W. Barth, C. Peters and A. van de Ven. Compact complex surfaces (Springer-Verlag, 1984)

[B] H.-J. BRAsch. Singularitäten von Modulräumen abelscher Flächen mit Polarisierung von Typ $(1, p)$. Thesis. Erlangen $(1994)$

[D] I. Dolgachev. Mirror symmetry for lattice polarized K3-surfaces. Duke e-prints alggeom/9502005

[EZ] M. Eichler and D. Zagier. The theory of Jacobi forms. Progress in Math. 55 (Birkhäuser $1985)$

[F] H.-G. Franke. Kurven in Hilbertschen Modulflächen und Humbertsche Flächen im Siegelraum. Bonner Math. Schriften 104 (1978)

[G1] V. Gritsenko. Modular forms and moduli spaces of abelian and K3 surfaces. St.Petersb. Math. Jour. 6:6 (1994), 65-102

[G2] V. GRITsEnko. Irrationality of the moduli spaces of polarized abelian surfaces. The International Mathematics Research Notices 6 (1994), pp. 235-243. In full form in Abelian Varieties. Proceedings of the Egloffstein conference 1993 (de Gruyter, 1995)

[GP] M. Gross and S. Popescu. Calabi Yau treefolds and moduli of abelian surfaces. Duke e-prints alg-geom $/ 9609001$

[Gu] L. GUTNiK. On extension of integral subgroups of some groups. Vestnik Leningradskogo Universiteta, Ser. Matemat. 12 (1957), 47-78.

[HL] K. HuleK and H. Lange. The Hilbert modular surface for the ideal $(\sqrt{ } 5)$ and the HorrocksMumford bundle. Math. Zeit. 198 (1988), 95-116.

[vdG] G. van Der GeER. Hilbert modular surfaces (Springer-Verlag, 1988).

[N1] V. Nikulin. Integral quadratic forms and some of its geometric applications. Izv. Akad. Nauk SSSR, Ser. Math. 43 (1979), 103-167.

[N2] V. Nikulin. Finite groups of automorphisms of Kähler K3 surfaces. Proc. Moscow Math. Society 38 (1980), 71-135.

[SZ $]$ N.-P. SkorupPa and D. ZAGier. Jacobi forms and a certain space of modular forms. Invent. Math. 94 (1988), 113-146.

Added in proof. 1. The assumption that $t$ is square free in Proposition 1.2 and Corollary 1.3 is unnecessary. Using arguments similar to those in $[\mathrm{Be}]$ one can show directly that the paramodular group $\Gamma_{t}$ is isomorphic to the corresponding orthogonal group for arbitrary $t$.

2. Theorem 1.5 gives a negative answer to a question raised by Shioda [S, question 5]. If $A$ is a general $(1, t)$-polarized abelian surface, then $A$ and its dual $A^{\prime}$ are not isomorphic (as projective surfaces), but their Kummer surfaces are isomorphic.

\section{REFERENCES}

[Be $]$ A. Beauville. Gèomètrie des surfaces K3: modules et périodes. Astèrisque 126 (1985), 99-128.

[S] T. Shioda. Some remarks on abelian surfaces. J. Fac. Sci. Univ. Tokyo. Sect IA 24 (1997), $11-21$. 